Homology, Homotopy and Applications, vol.15(1), 2013, pp.253-278

\title{
CELLULAR DECOMPOSITION AND FREE RESOLUTION FOR SPLIT METACYCLIC SPHERICAL SPACE FORMS
}

\author{
L.L. FÊMINA, A.P.T. GALVES, O. MANZOLI NETO AND \\ M. SPREAFICO
}

(communicated by Nigel Ray)

\begin{abstract}
Given a free isometric action of a split metacyclic group on odd dimensional sphere, we obtain an explicit finite cellular decomposition of the sphere equivariant with respect to the group action. A cell decomposition of the factor space and an explicit description of the associated cellular chain complex of modules over the integral group ring of the fundamental group follow. In particular, the construction provides a simple explicit 4-periodic free resolution for the split metacyclic groups.
\end{abstract}

\section{Introduction}

In [11] S. Tomoda and P. Zvenwgrovski studied the cohomology ring of the tridimensional spherical space forms. The basic idea is to produce an explicit resolution for the fundamental group $\pi$ of these spaces (the family of such groups is explicitly known). This is indeed a longstanding problem in algebraic topology, and we refer to the clear and complete description given in the first sections of [11] (see also $[\mathbf{1}]$ ). The abelian case, i.e., $\pi$ a cyclic group, was studied in seminal works of de Rham and Seifert. The next case to be investigated was $\pi$ a generalized quaternionic group. A resolution for these groups can be found, for example, in [2, Chap. XII, Sec. 7], but as observed in [11], no proof is presented. Proceeding along this line of investigation, Tomoda and Zvengrowski presented explicit resolutions for generalized quaternionic, binary tetrahedral, octahedral, and icosahedral groups, with explicit contracting homotopies. They also gave the ring structure of the remaining two families of groups, the split metacyclic groups and the $P_{8.3^{k}}$ (generalized binary tetrahedral) by using other techniques. For complete this investigation explicit resolutions for these two last families of groups is needed. This is the purpose of our project, which starts in this work where we study the case of the split metacyclic groups.

Our approach is based on the original idea of Swan [9]. Let a finite group $\pi$ act freely on a sphere $S^{n}$. Then, in order to obtain a resolution for $\pi$, it is sufficient to obtain a $\pi$-equivariant CW decomposition of $S^{n}$. Of course, the main problem in applying this approach is computational, and this is the reason why, after it was

Received October 23, 2012, revised February 27, 2012; published on May 28, 2013. 2000 Mathematics Subject Classification: 57M07, 57M10, 57Q10, 16E05, 18G10, 20J05, 20J06.

Key words and phrases: metacyclic group, fundamental domain, spherical space form.

Article available at http://intlpress.com/HHA/v15/n1/a13 and doi:10.4310/HHA.2013.v15.n1.a13

Copyright (C) 2013, International Press. Permission to copy for private use granted. 
successfully exploited for the cyclic groups, it was somehow abandoned. We were back to this technique in [7], where we studied the generalized quaternionic groups. In particular, we followed the clever geometric setting introduced by $\mathrm{F}$. Cohen in $[\mathbf{3}$, Chap. 9]. In that work, the cyclic group is considered, and a sophisticated description of the cellular complex was obtained using the join decomposition of a sphere in some spheres of lower dimension. We refer to that book for all the basic details of the construction. In [7], the ideas of Cohen were used, and after some substantial improvement of his technique, a cellular decomposition of the sphere $S^{n}$, equivariant with respect to the action of the generalized quaternionic groups, was obtained. We show in this work that this technique is, in fact, powerful enough in order to deal with another class of groups: the split metacyclic groups.

\section{Preliminaries and notations}

\subsection{Split metacyclic groups}

We denote by $D_{2^{t}(2 h+1)}$, with $t \geqslant 2$ and $h \geqslant 0$, the split metacyclic groups with presentation $[\mathbf{1 4}, 2.2]$

$$
D_{2^{t}(2 h+1)}=\left\langle x, y \mid x^{2 h+1}=y^{2^{t}}=1, y x y^{-1}=x^{-1}\right\rangle .
$$

The trivial case $t=h=0$ is not considered. The case $h=0$ gives a cyclic group $C_{2^{t}}$ $\left(\mathbb{Z} / 2^{t}\right.$ in multiplicative notation), the case $t=1$ gives the dihedral group $D_{2(2 h+1)}$ and the case $t=2$ gives the generalized quaternionic group $Q_{4(2 h+1)}([\mathbf{1 4}, 2.2]$ and $[\mathbf{1 1}$, Section 5.2]). All these cases have been studied elsewhere, so we will assume here that $t \geqslant 3$ and $h \geqslant 1$.

The split metacyclic groups also have the following semidirect product structure $[\mathbf{1 1}$, Section 5.2]:

$$
D_{2^{t}(2 h+1)} \cong C_{2 h+1} \rtimes_{\varphi} C_{2^{t}},
$$

with $\varphi: C_{2^{t}} \rightarrow \operatorname{Aut}\left(C_{2 h+1}\right)$, defined by $\varphi(1)=-1$. This is related with the split short exact sequence

$$
1 \longrightarrow C_{2 h+1} \stackrel{i}{\longrightarrow} D_{2^{t}}(2 h+1) \underset{S}{\stackrel{p}{\longleftrightarrow}} C_{2^{t}} \longrightarrow 1,
$$

where $i$ is an inclusion onto the normal subgroup generated by $i(x)=x, p(y)=y$ (the generator of $\left.C_{2^{t}}\right), p(x)=1$, and the splitting maps is $s(y)=y$. Note also that $\left(D_{2^{t}(2 h+1)}\right)_{\mathrm{ab}}=C_{2^{t}}$.

Remark 2.1. The elements of $D_{2^{t}(2 h+1)}$ can be written as follows:

$$
D_{2^{t}(2 h+1)}=\left\{1, x, x^{2}, \ldots, x^{2 h}, y, x y, \ldots, x^{2 h} y, \ldots, y^{2^{t}-1}, x y^{2^{t}-1}, \ldots, x^{2 h} y^{2^{t}-1}\right\} .
$$

\subsection{Matrix representation of group actions}

Since notation will have a relevant bearing on the following developments, we spend a few lines recalling the notation adopted for the matrices representing the groups actions, according to [13]. Let $V, W$ be fixed finite dimensional vector spaces over a field $\mathbb{F}$ of characteristic zero, with $\operatorname{dim} V=n, \operatorname{dim} W=k$. Denote by $V^{\dagger}=$ $\operatorname{Hom}_{\mathbb{F}}(V, \mathbb{F})$ the dual space. Let $e=\left\{e_{1}, \ldots, e_{n}\right\}$ and $b=\left\{b_{1}, \ldots, b_{k}\right\}$ be given bases 
of $V$ and $W$, respectively. Let $M(n, k ; \mathbb{F})$ denote the set of matrices with $n$ lines and $k$ columns, with standard matrix product. To each homomorphism $\varphi \in \operatorname{Hom}_{\mathbb{F}}(V, W)$ we associate the matrix $M(\varphi) \in M(n, k ; \mathbb{F})$ defined by $M(\varphi)=\left(\varphi_{j, l}=b_{k}^{\dagger}\left(\varphi\left(e_{j}\right)\right)\right)$, where $\left(\varphi_{j, l}\right)$ denotes the matrix with entry $\varphi_{j, l}$ at line $j$ and column $l$, i.e.,

$$
\varphi\left(e_{j}\right)=\sum_{l=1}^{k} \varphi_{j, l} b_{l} .
$$

The application $M: \operatorname{Hom}_{\mathbb{F}}(V, V) \rightarrow M(n, n ; \mathbb{F})$ is a homomorphism of groups, if we equip $\operatorname{Hom}_{F}(V, V)$ with the product $\varphi_{1} \cdot \varphi_{2}=\varphi_{2} \varphi_{1}$ (juxtaposition denotes composition), i.e., $M\left(\varphi_{1} \cdot \varphi_{2}\right)=M\left(\varphi_{1}\right) M\left(\varphi_{2}\right)$.

Next, let $\rho: G \rightarrow \operatorname{Aut}_{\mathrm{F}}(V)$ be a group representation. We identify representations with right $\pi$ actions on $V$, and hence we use the multiplication - in $\operatorname{Aut}_{\mathrm{F}}(V)$, namely $\rho\left(g_{1}\right) \cdot \rho\left(g_{2}\right)=\rho\left(g_{2}\right) \rho\left(g_{1}\right)$. Then, the composite $M \rho: G \rightarrow M(n ; \mathbb{F})$ is a group homomorphism: $M \rho\left(g_{1} g_{2}\right)=M \rho\left(g_{1}\right) M \rho\left(g_{2}\right)$.

We conclude by observing that we will, in general, denote by juxtaposition the action of a group element $g$ on a vector $v$, whenever the representation is clear. We will use the following equivalent notations:

$$
\left.v g=\rho(g)(v)=\left(v_{1} \cdots v_{n}\right)\right) M \rho(g) .
$$

\subsection{Free actions on spheres}

The groups $D_{2^{t}(2 h+1)}$ are groups of type I according to the table of Theorem 6.1.11 of J. Wolf [13], with $A=x, B=y, m=2 h+1, n=2^{t}, r=-1, d=2, n^{\prime}=2^{t-1}$ and satisfy any of the three conditions given below the table (where $d$ is defined, $n^{\prime}$ is defined in [13, Theorem 5.5.1]). Then, according to Wolf [13, Theorem 5.5.6] the irreducible faithful complex representations of $D_{2^{t}(2 h+1)}$ have degree 2, are fixed point free and are explicitly given by (note we use transposed notation with respect to [13])

$$
\begin{aligned}
& \pi_{k, l}(x)=M \rho_{k, l}(x)=\left(\begin{array}{cc}
\mathrm{e}^{\frac{2 \pi k i}{2 h+1}} & 0 \\
0 & \mathrm{e}^{-\frac{2 \pi k i}{2 h+1}}
\end{array}\right), \\
& \pi_{k, l}(y)=M \rho_{k, l}(y)=\left(\begin{array}{cc}
0 & \mathrm{e}^{\frac{\pi l i}{2^{t-2}}} \\
1 & 0
\end{array}\right),
\end{aligned}
$$

where $k$ and $l$ are integers with $(k, 2 h+1)=(l, 2)=1$. By the same theorem, all possible automorphisms of $D_{2^{t}(2 h+1)}$ are of the form

$$
\varphi_{a, b, c}:\left\{\begin{array}{l}
x \mapsto x^{a}, \\
y \mapsto y^{b} x^{c},
\end{array}\right.
$$

with integers $a, b, c$ with $(a, 2 h+1)=(b, 2)=1, b=1 \bmod 2$. Moreover, the composition $\pi_{k, l} \varphi_{a, b, c}$ is equivalent to $\pi_{a k, b l}$, and $\pi_{k, l}$ is equivalent to $\pi_{k^{\prime}, l^{\prime}}$ if and only if $l^{\prime}=l \bmod 2^{t-1}$ and $k^{\prime}= \pm k \bmod (2 h+1)$.

These facts allow us to determine the following set of representative representations for the different classes of equivalent representations: 
Lemma 2.2. The non-equivalent irreducible complex representations of $D_{2^{t}(2 h+1)}$ are

$$
\begin{aligned}
& \pi_{k, l}: D_{2^{t}(2 h+1)} \rightarrow U(2, \mathbb{C}), \\
& \pi_{k, l}:\left\{\begin{array}{l}
x \mapsto\left(\begin{array}{cc}
\mathrm{e}^{\frac{2 \pi k i}{2 h+1}} & 0 \\
0 & \mathrm{e}^{-\frac{2 \pi k i}{2 h+1}}
\end{array}\right), \\
y \mapsto\left(\begin{array}{cc}
0 & \mathrm{e}^{\frac{\pi l i}{2^{t-2}}} \\
1 & 0,
\end{array}\right)
\end{array}\right.
\end{aligned}
$$

with $k=1,2, \ldots, h, l=1,3, \ldots, 2^{t-1}-1$ and $(k, 2 h+1)=(l, 2)=1$. All these representations are fixed point free.

Next, consider the actions of $D_{2^{t}(2 h+1)}$ on odd spheres $S^{4 n-1}$. By [13, 7.4], all possible actions are direct sums of the $\pi_{k, l}$. Namely, let $\mathbf{k}=\left(k_{1}, \ldots, k_{n}\right)$ be a vector of integers prime to $(2 h+1)$ and let $\mathbf{l}=\left(l_{1}, \ldots, l_{n}\right)$ be a vector of integers prime to 2. Then define the representation

$$
\pi_{\mathbf{k} ; \mathbf{l}}=\pi_{k_{1}, l_{1}} \oplus \cdots \oplus \pi_{k_{n}, l_{n}}: D_{2^{t}(2 h+1)} \rightarrow U(2 n, \mathbb{C}),
$$

where $\pi_{k_{j}, l_{j}}: D_{2^{t}(2 h+1)} \rightarrow U(2, \mathbb{C})$ have the following form:

$$
\begin{aligned}
& \pi_{k_{j}, l_{j}}(x)=\left(\begin{array}{cc}
\mathrm{e}^{\frac{2 \pi k_{j} i}{2 h+1}} & 0 \\
0 & \mathrm{e}^{-\frac{2 \pi k_{j} i}{2 h+1}}
\end{array}\right)=\left(\begin{array}{cc}
\zeta^{k_{j}} & 0 \\
0 & \zeta^{-k_{j}}
\end{array}\right), \\
& \pi_{k_{j}, l_{j}}(y)=\left(\begin{array}{cc}
0 & \mathrm{e}^{\frac{\pi l_{j} i}{2^{t-2}}} \\
1 & 0
\end{array}\right)=\left(\begin{array}{cc}
0 & \xi^{l_{j}} \\
1 & 0
\end{array}\right) .
\end{aligned}
$$

Remark 2.3. Note that all the real representations associated to the representations in Lemma 2.2 are orthogonal.

We call the factor $S^{4 n-1} / \pi_{\mathbf{k} ; \mathbf{l}}\left(D_{2^{t}(2 h+1)}\right)$ a split metacyclic spherical space form, and we denote it by $\mathbf{S}_{2^{t}(2 h+1) ; \mathbf{k} ; \mathbf{l}}^{4 n-1}$. It is not difficult to see that the following representatives of homeomorphic classes of split metacyclic spherical space forms can be chosen.

The proof of the following proposition is straightforward and will be omitted (compare with [8, Section 12] and [7, Lemma 2]).

Proposition 2.4. Any split metacyclic spherical space form is homeomorphic to one of type $\mathbf{S}_{2^{t}(2 h+1) ; k_{1}, \ldots, k_{n} ; l_{1}, \ldots, l_{n}}^{4 n-1}$, with $1 \leqslant k_{1} \leqslant k_{2} \leqslant \cdots \leqslant k_{n}<h, 1 \leqslant l_{1} \leqslant l_{2} \leqslant \cdots<$ $2^{t-1}$ and $(k, 2 h+1)=(l, 2)=1$.

\subsection{Curved join}

Given two unitary complex numbers $z_{1}, z_{2} \in \mathbb{C}$, consider the ordered pair $\left(z_{1}, z_{2}\right)$ in $\mathbb{C} \times \mathbb{C}=\mathbb{R}^{4}$. Since there exists only one plane in $\mathbb{R}^{4}$ through the origin, $z_{1}$ and $z_{2}$, we can take the oriented arc from $z_{1}$ to $z_{2}$ in the unitary circle on this plane and with length $\pi / 2$. We denote this arc by $z_{1} * z_{2}$ and its end points by $z_{1} * \emptyset$ and $\emptyset * z_{2}$. If the two points, say $w_{1} \neq w_{2}$, lay on the same circle, then we use the notation 
$\left[w_{1}, w_{2}\right]$ for the oriented arc from $w_{1}$ to $w_{2}$. For any two subsets $Z_{1}$ and $Z_{2}$, with $Z_{1} \times Z_{2} \subset S^{1} \times S^{1} \subset \mathbb{C} \times \mathbb{C}$, we define their curved join by

$$
Z_{1} * Z_{2}=\left\{z_{1} * z_{2} \mid z_{1} \in Z_{1}, z_{2} \in Z_{2}\right\} .
$$

For example, $S^{1} * S^{1}=S^{3}$. This process generalizes as follows: Identify $\mathbb{C}^{m}$ with $\mathbb{R}^{2 m}$, and, given the standard orthonormal basis $\left\{e_{1}, \ldots, e_{2 m}\right\}$ of $\mathbb{R}^{2 m}$, for each $r \neq s$, denote by $\Pi_{r, s}$ the plane generated by $\left\{e_{r}, e_{s}\right\}$. Suppose $\Pi_{r_{1}, s_{1}} \cap \Pi_{r_{2}, s_{2}}=\{0\}$. Let $Z_{1}$ and $Z_{2}$ be subsets of the unit circles of $\Pi_{r_{1}, s_{1}}$ and $\Pi_{r_{2}, s_{2}}$, respectively. Then the curved join $Z_{1} * Z_{2}$ is well defined by equation (2.1). In particular, we denote by $\Sigma_{l}$ the unit circle laying in the $l$-th complex hyperplane of $\mathbb{C}^{2 n}$. Then, we have an homeomorphism of the iterated curved join

$$
S^{4 n-1}=\Sigma_{1} * \cdots * \Sigma_{2 n} .
$$

For further use, it is convenient to identify the basis vector with their final points and to use the $e_{j}$ to denote the points in $S^{4 n-1}$. Then, for example, the canonical basis of $\mathbb{R}^{4}$ in $S^{3}$ is

$$
\begin{array}{ll}
e_{1}=(1,0,0,0)=1 * \emptyset, & e_{2}=(0,1,0,0)=i * \emptyset, \\
e_{3}=(0,0,1,0)=\emptyset * 1, & e_{4}=(0,0,0,1)=\emptyset * i .
\end{array}
$$

It will be also useful to identify $S^{3}=S^{1} * S^{1}$ with the union of two solid torus along the common boundary. Each $S^{1}$ is then the central circle inside one of the solid torus. We can then represent the two half spheres $S^{1} * S_{ \pm}^{1}$ (where $S_{ \pm}^{1}$ denotes the north/south hemisphere of $S^{1}$ ) as in Figure 1, where the framing is given by the geodesic lines joining the end points of the basic vectors $e_{j}$ (see [3, Section 26] for details).
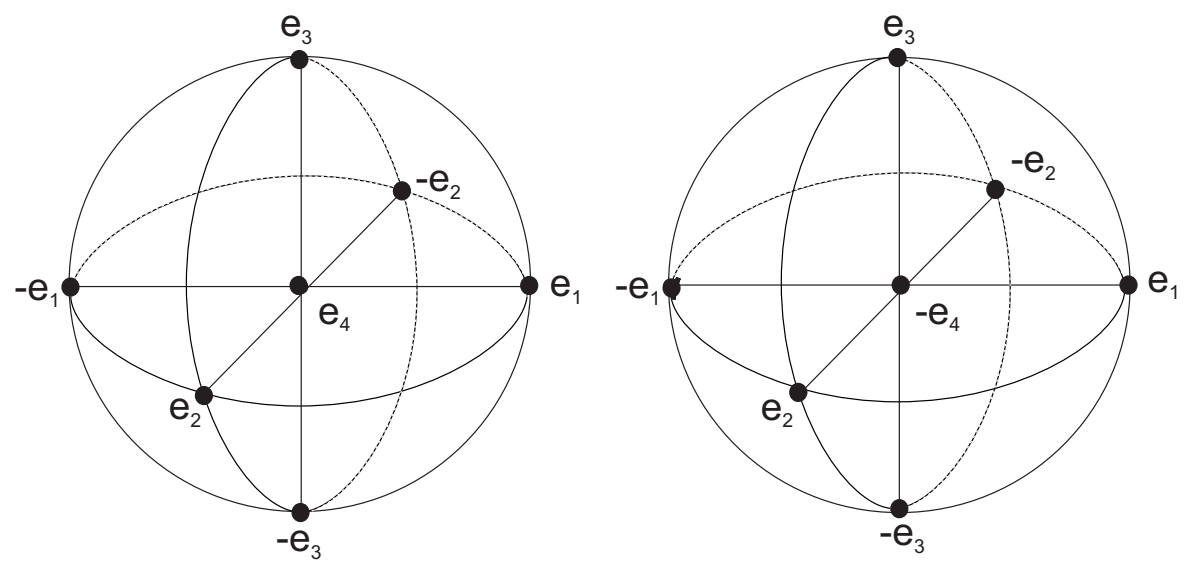

Figure 1

It is clear that the curved join is homeomorphic to the usual join:

$$
J(X, Y)=\frac{X \times I \times Y}{(\{x\} \times 0 \times Y) \cup(X \times 1 \times\{y\})} .
$$

However, the usual join $J(X, Y)$ and the curved join $X * Y$ are not isometric. The 
metric of the curved join is the metric of the sphere, and the segments are segments of geodesic. In particular, this is fundamental when we describe the natural action appearing definition of the spherical space forms. More precisely, let $\pi$ be a finite group acting freely and orthogonally on a sphere $S^{n}$, and let $h$ be a positive integer. Then, there is a natural action of $\pi$ on $S^{h(n+1)-1}$ defined by

$$
\begin{aligned}
\left(S^{h(n+1)-1} \subset\left(\mathbb{R}^{n+1}\right)^{h}\right) \times G & \rightarrow S^{h(n+1)-1} \subset\left(\mathbb{R}^{n+1}\right)^{h}, \\
\left(\left(x_{1}, \ldots, x_{h}\right), g\right) & \mapsto\left(g x_{1}, \ldots, g x_{h}\right) .
\end{aligned}
$$

This action coincides with the action

$$
\begin{aligned}
\left(S^{h(n+1)-1}=S^{(h-1)(n+1)-1} * S^{n}\right) \times G & \rightarrow S^{h(n+1)-1}=S^{(h-1)(n+1)-1} * S^{n}, \\
((x, t, y), g) & \mapsto(g x, t, g y),
\end{aligned}
$$

where the join is the curved join.

We conclude with a technical result.

Lemma 2.5. We have the following formula for the boundary:

$$
\partial(X * Y)=\partial(X) * Y+(-1)^{\operatorname{dim} X+1} X * \partial(Y) .
$$

\section{The tridimensional case}

In this section we present the two major technical results of this work: in the first subsection we describe the fundamental domain $\mathcal{F}$ for the action of $D_{2^{t}(2 h+1)}$ via the representation $\pi_{k, l}$ on the tridimensional sphere $S^{3}$, and in the second subsection we achieve a $\mathrm{CW}$ decomposition of $\mathcal{F}$ by first obtaining an equivariant $\mathrm{CW}$ decomposition of $S^{3}$. Both the fundamental domain and the CW decomposition for the higher dimensional cases will follow by those for the tridimensional case and some general results, and will be presented in Section 4 .

\subsection{The fundamental domain}

In order to deal with the fundamental domain, we will use the approach and the notation introduced in [7]. First, recall that if $G$ is a finite group acting on a space $\mathbf{S}$, then a fundamental domain of the action of $\pi$ on $\mathbf{S}$ is a connected closed subset $\mathcal{F}$ of $\mathbf{S}$ such that $\mathbf{S}=\bigcup_{g} g \mathcal{F}$ and $g \mathcal{F} \cap g^{\prime} \mathcal{F}$ has empty interior for all $g \neq g^{\prime} \in G$. The first important difference with respect to the case of the quaternion studied in [7] is that in the present case the fundamental domain can change when we change the representation. We start by observing that the image of the cyclic subgroup generated by $x, \pi_{k, l}(\langle x\rangle)$ is independent on $k$ and $l$, while the image of the subgroup generated by $y, \pi_{k, l}(\langle y\rangle)$ is independent on $k$ but depends on $l$. This means that the fundamental domain cannot change when we change the parameter $k$, but, in principle, depends on $l$. Thus, in order to determine the fundamental domain, we can fix $k=1$ in the representation.

Next, consider the curved join description of $S^{3}=S^{1} * S^{1}$ given in the previous section. Let us introduce the following minimal angle:

$$
\varepsilon=\frac{4 \pi}{\left|D_{2^{t}(2 h+1)}\right|}=\frac{2 \pi}{2^{t-1}(2 h+1)} .
$$


Since $l$ is odd it is prime to $2^{t-1}$, and we have $2^{t-2}$ distinct values for $l$. So for each fixed $l$ we have $\frac{2^{t-1}(2 h+1)}{2^{t-2}}=2(2 h+1)$ possible different fundamental domains in $S^{3}$. Let us introduce some further notation. We denote the final point of the basis vector $e_{k}$ by the same notation $e_{k}$. Recall from Section 2.4 that this is the point of the circle $\Sigma_{k}$ with real coordinates $(\underbrace{0, \ldots, 0,1}_{k}, \ldots 0)$ in $\mathbb{R}^{4 n}$. Let $A_{1}$ denote the arc of $\Sigma_{1}$ of length $\frac{\theta_{1}}{2}=2^{t-2} \varepsilon$ starting at $e_{2}$ in the positive direction, and let $A_{2}$ denote the arc of $\Sigma_{2}$ of length $\theta_{2}=(2 h+1) \varepsilon$ starting at $e_{4}$ in the negative direction; see Figure 2 (recall $\zeta=\mathrm{e}^{\frac{2 \pi}{2 h+1}}, \xi=\mathrm{e}^{\frac{2 \pi}{2^{t-1}}}$ ):

$$
A_{1}=\left[i, \zeta^{\frac{1}{2}} i\right] * \emptyset=e_{2} * \zeta^{\frac{1}{2}} e_{2}, \quad A_{2}=\emptyset *[i, \xi i]=e_{4} * \xi e_{4} .
$$

Next, we describe the action of the group generators $x$ and $y$ in the representation $\pi_{1,1}$. Let $\alpha_{j}=\left[z_{j}, w_{j}\right]$ be an arc of $\Sigma_{j}$, and let $R(\theta)$ denote the rotation of angle $\theta$. Then,

$$
\begin{aligned}
& \rho_{1,1}(x)\left(\alpha_{1} * \alpha_{2}\right)=R\left(\theta_{1}\right)\left(\alpha_{1}\right) * R\left(-\theta_{1}\right)\left(\alpha_{2}\right), \\
& \rho_{1,1}(y)\left(\alpha_{1} * \alpha_{2}\right)=R\left(\theta_{2}\right)\left(\alpha_{2}\right) * \alpha_{1} .
\end{aligned}
$$

Observe that the notation $\alpha_{1} * \alpha_{2}$ means the arc $\alpha_{1}=\left[z_{1}, w_{1}\right]$ in $\Sigma_{1}$, starting at the point $z_{1}$ of $\Sigma_{1}$ and ending at the point $w_{1}$ of $\Sigma_{1}$, joins the $\operatorname{arc} \alpha_{2}=\left[z_{2}, w_{2}\right]$ in $\Sigma_{2}$, starting at the point $z_{2}$ of $\Sigma_{2}$ and ending at the point $w_{2}$ of $\Sigma_{2}$.

In more detail, we have:

(X1) $\rho_{1,1}(x)\left(\alpha_{1} * \emptyset\right)=\left[R\left(\theta_{1}\right)\left(z_{1}\right), R\left(\theta_{1}\right)\left(w_{1}\right)\right] * \emptyset$,

i.e., $x$ rotates $\alpha_{1}$ by $2^{t-1} \varepsilon$ in the positive sense in $\Sigma_{1}$.

(X2) $\rho_{1,1}(x)\left(\emptyset * \alpha_{2}\right)=\emptyset *\left[R\left(-\theta_{1}\right)\left(z_{2}\right), R\left(-\theta_{1}\right)\left(w_{2}\right)\right]$,

i.e., $x$ rotates $\alpha_{2}$ by $2^{t-1} \varepsilon$ in the negative sense in $\Sigma_{2}$.

(Y1) $\rho_{1,1}(y)\left(\alpha_{1} * \emptyset\right)=\emptyset *\left[z_{1}, w_{1}\right]$,

i.e., $y$ moves $\alpha_{1}$ from $\Sigma_{1}$ into $\Sigma_{2}$.

(Y2) $\rho_{1,1}(y)\left(\emptyset * \alpha_{2}\right)=\left[R\left(\theta_{2}\right)\left(z_{2}\right), R\left(\theta_{2}\right)\left(w_{2}\right)\right] * \emptyset$,

i.e., $y$ moves $\alpha_{2}$ from $\Sigma_{2}$ into $\Sigma_{1}$ and rotates it by $(2 h+1) \varepsilon$ in the positive sense in $\Sigma_{1}$.

The proof follows by definition and Lemma 2.2, and the fact that the action of the group generators on the standard basis vectors $b_{1}=(1,0)$ and $b_{2}=(0,1)$ of $\mathbb{C}^{2}$ are

$$
\begin{array}{llrl}
\rho_{1,1}(x)\left(b_{1}\right) & =\zeta^{\frac{1}{2}} b_{1}, & \rho_{1,1}(x)\left(b_{2}\right) & =\bar{\zeta}^{\frac{1}{2}} b_{2}, \\
\rho_{1,1}(y)\left(b_{1}\right) & =b_{2}, & \rho_{1,1}(y)\left(b_{2}\right) & =\xi b_{1} .
\end{array}
$$

Remark 3.1. Note that

$$
\begin{aligned}
\rho_{1,1}\left(x^{a}\right)\left(\alpha_{1} * \alpha_{2}\right) & =R\left(a \theta_{1}\right)\left(\alpha_{1}\right) * R\left(-a \theta_{2}\right)\left(\alpha_{2}\right), \\
\rho_{1,1}\left(y^{2 b}\right)\left(\alpha_{1} * \alpha_{2}\right) & =R\left(b \theta_{2}\right)\left(\alpha_{1}\right) * R\left(b \theta_{2}\right)\left(\alpha_{2}\right), \\
\rho_{1,1}\left(y^{2 b+1}\right)\left(\alpha_{1} * \alpha_{2}\right) & =R\left((b+1) \theta_{2}\right)\left(\alpha_{2}\right) * R\left(b \theta_{2}\right)\left(\alpha_{1}\right) .
\end{aligned}
$$

The main purpose of this section is to prove the following result:

Proposition 3.2. A fundamental domain for the action of the group $D_{2^{t}(2 h+1)}$ on $S^{3}$ via the representation $\pi_{1,1}$ is $\mathcal{F}_{1,1}=A_{1} * A_{2}=\left[i, \zeta^{\frac{1}{2}} i\right] *[i, \xi i]$. 


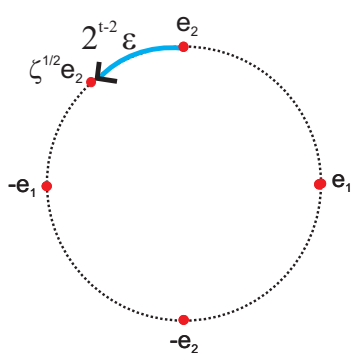

The $\operatorname{arc} \mathrm{A}_{1}$

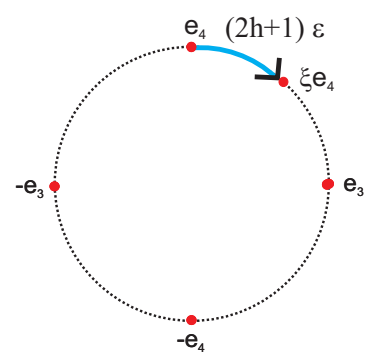

The $\operatorname{arc} \mathrm{A}_{2}$

Figure 2

In Figure 3 we represent the fundamental domain $\mathcal{F}_{1,1}$ using the description of $S^{3}$ introduced at the end of Section 2.4. In the figure the fundamental domain is illustrated as embedded in $S^{3}$, framed by the geodesic line joining the end points of the basis vectors $e_{j}$. The end points of the arcs $A_{j}$ are also displayed. In Figure 4 the fundamental domain appears without the framing. This will be useful in the following lemmas in order to describe the cell decomposition of the fundamental domain:
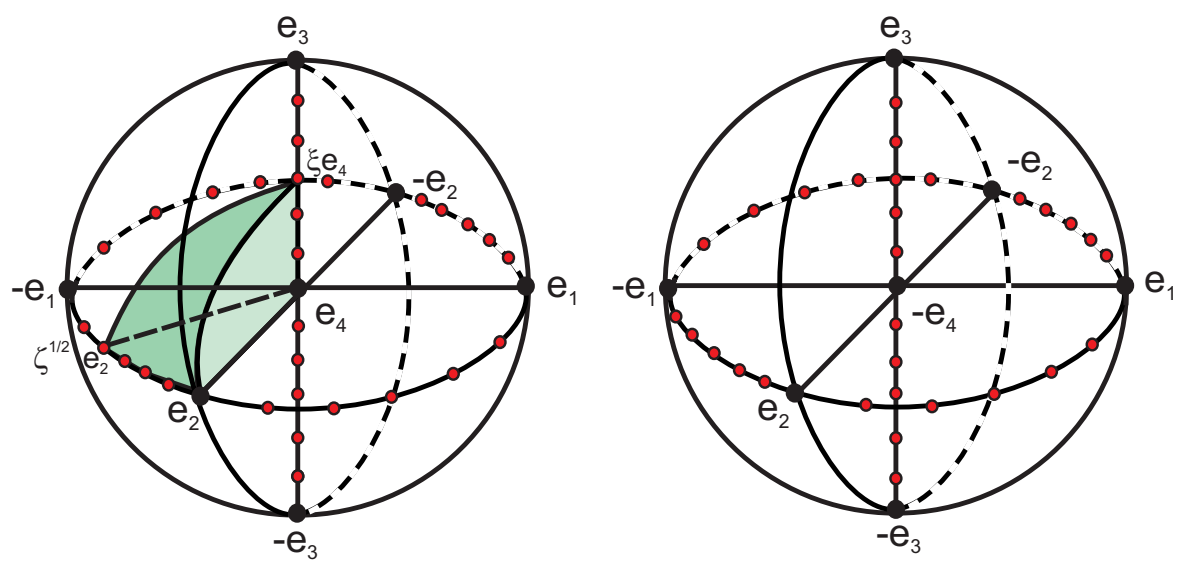

Figure 3

The proof of Proposition 3.2 is based on the following lemmas:

Lemma 3.3. Consider $P * Q$ and $P^{\prime} * Q^{\prime}$ as two subsets in $S^{1} * S^{1}, \operatorname{Int}(P * Q) \cap$ $\operatorname{Int}\left(P^{\prime} * Q^{\prime}\right) \neq \emptyset$ if and only if $\operatorname{Int}(P) \cap \operatorname{Int}\left(P^{\prime}\right) \neq \emptyset$ and $\operatorname{Int}(Q) \cap \operatorname{Int}\left(Q^{\prime}\right) \neq \emptyset$.

Lemma 3.4. The rotations on the two circles $\Sigma_{j}$ determined by the action of the group elements on $\mathcal{F}_{1,1}$ in the representation $\rho_{1,1}$ satisfy the following bounds:

1. For all $a=1,2, \ldots, 2 h, \rho_{1,1}\left(x^{a}\right)\left(A_{1} * A_{2}\right)=R\left(a \theta_{1}\right)\left(A_{1}\right) * R\left(-a \theta_{1}\right)\left(A_{2}\right)$, with $0 \leqslant \theta_{1}<2 \pi$, i.e., the rotations determined by $x^{a}$ on $\Sigma_{1}$ and $\Sigma_{2}$ are less than $2 \pi$. 


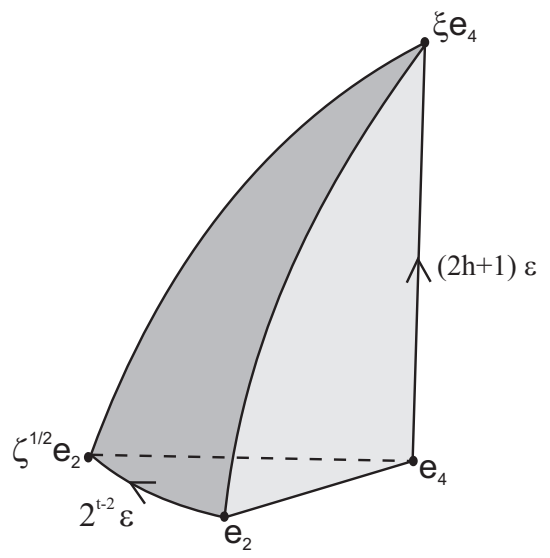

Figure 4

2. For all $b=1,2, \ldots, 2^{t-1}$,

$$
\rho_{1,1}\left(y^{2 b-1}\right)\left(A_{1} * A_{2}\right)=R\left(b \theta_{2}\right)\left(A_{2}\right) * R\left((b-1) \theta_{2}\right)\left(A_{1}\right),
$$

with $0 \leqslant b \theta_{2} \leqslant 2 \pi$ e $0 \leqslant(b-1) \theta_{2}<2 \pi$, i.e., the rotation determined by $y^{2 b-1}$ on $\Sigma_{1}$ is less than or equal to $2 \pi$, and that on $\Sigma_{2}$ is less than $2 \pi$.

3. For all $a=1,2, \ldots, 2 h, b=1,2, \ldots, 2^{t-1}$,

$$
\rho_{1,1,}\left(x^{a} y^{2 b-1}\right)\left(A_{1} * A_{2}\right)=R\left(a \theta_{1}+b \theta_{2}\right)\left(A_{2}\right) * R\left(-a \theta_{1}+(b-1) \theta_{2}\right)\left(A_{1}\right),
$$

with $0 \leqslant a \theta_{1}+b \theta_{2}<4 \pi$ and $0 \leqslant-a \theta_{1}+(b-1) \theta_{2}<2 \pi$, i.e., the rotation determined by $x^{a} y^{2 b-1}$ on $\Sigma_{1}$ is less than $4 \pi$, and that on $\Sigma_{2}$ is less than $2 \pi$.

4. For all $b=1,2, \ldots, 2^{t-1}-1, \rho_{1,1}\left(y^{2 b}\right)\left(A_{1} * A_{2}\right)=R\left(b \theta_{2}\right)\left(A_{1}\right) * R\left(b \theta_{2}\right)\left(A_{2}\right)$, with $0 \leqslant b \theta_{2}<2 \pi$, i.e., the rotations determined by $y^{2 b}$ on $\Sigma_{1}$ and $\Sigma_{2}$ are less than $2 \pi$.

5. For all $a=1,2, \ldots, 2 h, b=1,2, \ldots, 2^{t-1}-1, \rho_{1,1}\left(x^{a} y^{2 b}\right)\left(A_{1} * A_{2}\right)=R\left(a \theta_{1}+\right.$ $\left.b \theta_{2}\right)\left(A_{1}\right) * R\left(-a \theta_{1}+b \theta_{2}\right)\left(A_{2}\right)$, with $0 \leqslant a \theta_{1}+b \theta_{2}<4 \pi$ and $0 \leqslant-a \theta_{1}+b \theta_{2}<$ $2 \pi$, i.e., the rotation determined by $x^{a} y^{2 b}$ on $\Sigma_{1}$ is less than $4 \pi$, and that on $\Sigma_{2}$ is less than $2 \pi$.

Proof. This follows by lengthy direct verification. We show the proof for one illustrative case, and we refer to $[\mathbf{6}, 2.2 .7]$ for the complete details. We prove case (3). The rotation on $\Sigma_{1}$ determined by the action of $x^{a} y^{2 b-1}$ on $\mathcal{F}_{1,1}$ consists of rotating the $\operatorname{arc} A_{1}$ by an arc of length $\left(2^{t-1} a+(2 h+1) b\right) \epsilon$ in the counterclockwise sense by (X1) and (Y2). Since the maximum values of $a$ and $b$ are $\max (a)=2 h$ and $\max (b)=2^{t-1}$, the maximum rotation is

$$
\left(2^{t-1} \max (a)+(2 h+1) \max (b)\right) \epsilon=\left(2^{t} h+2^{t-1}(2 h+1)\right) \epsilon .
$$

Since $2^{t} h<2^{t-1}(2 h+1)$, it follows that $2^{t} h+2^{t-1}(2 h+1)<2\left(2^{t-1}\right)(2 h+1)$. Whence, the maximum rotation of $A_{1}$ will be less that $4 \pi\left(\right.$ recall $\left.2^{t-1}(2 h+1) \epsilon=2 \pi\right)$. 
The rotation on $\Sigma_{2}$ determined by the action of $x^{a} y^{2 b-1}$ on $\mathcal{F}_{1,1}$ consists of rotating the arc $A_{2}$ by an arc of length $\left(-2^{t-1} a+(2 h+1)(b-1)\right) \epsilon$ in the counterclockwise sense by (X2) and (Y1). Taking the maximum and minimum values for $a$ and $b$ $(\min (b)=1)$, the maximum rotation is

$$
\left(-2^{t-1} \min (a)+(2 h+1)(\max (b)-1)\right) \epsilon=\left(\left(2^{t}-2\right) h-1\right) \epsilon<2 \pi .
$$

Lemma 3.5. The following affirmations are true:

1. For all $a=1,2, \ldots, 2 h$, $\operatorname{Int}\left(\rho_{1,1}\left(x^{a}\right)\left(\mathcal{F}_{1,1}\right)\right) \cap \operatorname{Int}\left(\mathcal{F}_{1,1}\right)=\emptyset$.

2. For all $b=1,2, \ldots, 2^{t-1}-1$, $\operatorname{Int}\left(\rho_{1,1}\left(y^{2 b}\right)\left(\mathcal{F}_{1,1}\right)\right) \cap \operatorname{Int}\left(\mathcal{F}_{1,1}\right)=\emptyset$.

3. For all $b=1,2, \ldots, 2^{t-1}, \operatorname{Int}\left(\rho_{1,1}\left(y^{2 b-1}\right)\left(\mathcal{F}_{1,1}\right)\right) \cap \operatorname{Int}\left(\mathcal{F}_{1,1}\right)=\emptyset$.

4. For all $a=1,2, \ldots, 2 h$, and $b=1,2, \ldots, 2^{t-1}-1$,

$$
\operatorname{Int}\left(\rho_{1,1}\left(x^{a} y^{2 b}\right)\left(\mathcal{F}_{1,1}\right)\right) \cap \operatorname{Int}\left(\mathcal{F}_{1,1}\right)=\emptyset .
$$

5. For all $a=1,2, \ldots, 2 h$, and $b=1,2, \ldots, 2^{t-1}$,

$$
\operatorname{Int}\left(\rho_{1,1}\left(x^{a} y^{2 b-1}\right)\left(\mathcal{F}_{1,1}\right)\right) \cap \operatorname{Int}\left(\mathcal{F}_{1,1}\right)=\emptyset .
$$

Proof. The method of proving these facts is always the same: we first show that the geometric condition is equivalent to some equations in $\mathbb{Z}$, and then that such equations have no solutions, by contradiction. We outline the proof here; all details can be found in the proof of Proposition 2.2.8 of [6].

1. In order to prove Lemma 3.5 first recall that the action of $x$ does not interchange the points of the two planes of $\Sigma_{1}$ and $\Sigma_{2}$, and the rotation determined by $x^{a}$ is $2^{t-1} a \epsilon$. Then, by direct analysis of the geometric description and by Lemmas 3.3 and 3.4, $\operatorname{Int}\left(\rho_{1,1}\left(x^{a}\right)\left(\mathcal{F}_{1,1}\right)\right) \cap \operatorname{Int}\left(\mathcal{F}_{1,1}\right) \cap \Sigma_{1}=\operatorname{Int}\left(\rho_{1,1}\left(x^{a}\right)\left(A_{1}\right)\right) \cap \operatorname{Int}\left(A_{1}\right)$ can be non-empty only if the following condition is fulfilled:

(a1) $2^{t-1} a=c$, with $c=1,2, \ldots, 2^{t-2}-1$.

If this is the case, since $\max (c)=2^{t-2}-1$, then $a \leqslant \frac{2^{t-2}-1}{2^{t-1}}<1$ is not an integer.

2. The proof is analogous to the previous one, given that $\operatorname{Int}\left(\rho_{1,1}\left(y^{2 b}\right) \mathcal{F}_{1,1}\right) \cap$ $\operatorname{Int}\left(\mathcal{F}_{1,1}\right) \cap \Sigma_{2}=\operatorname{Int}\left(\rho_{1,1}\left(y^{2 b}\right)\left(A_{2}\right)\right) \cap \operatorname{Int}\left(A_{2}\right)$ can be non-empty only if:

(b1) $(2 h+1) b=2^{t-1}(2 h+1)-d$, with $d=1,2, \ldots, 2 h$.

3. In order to prove this affirmation, we need a combination of the previous conditions, since now the action of the group elements can interchange the planes. First, we find that $\operatorname{Int}\left(\rho_{1,1}\left(y^{2 b-1}\right) \mathcal{F}_{1,1}\right) \cap \operatorname{Int}\left(\mathcal{F}_{1,1}\right) \cap \Sigma_{1}$ can be non-empty only if:

(a1) $(2 h+1) b=c, c=1,2, \ldots, 2 h+2$,

while $\operatorname{Int}\left(\rho_{1,1}\left(y^{2 b-1}\right)\left(\mathcal{F}_{1,1}\right)\right) \cap \operatorname{Int}\left(\mathcal{F}_{1,1}\right) \cap \Sigma_{2}$ can be non-empty only if one of the following two conditions is fulfilled:

(b1) $(2 h+1)(b-1)=-d, d=1,2, \ldots, 2 h+2$,

(b2) $(2 h+1)(b-1)=2^{t-1}(2 h+1)-d, d=1,2, \ldots, 2 h+2$.

We show that none of the combinations $(a 1)$ and $(b k), k=1,2$, is possible. Assume (a1) and (b1) hold. Then there are integers $a$ and $b$ such that 


$$
\begin{cases}(a 1) & (2 h+1) b=c \\ (b 1) & (2 h+1)(b-1)=-d .\end{cases}
$$

Since $1 \leqslant c, d \leqslant 2 h+2$, and $b$ is an integer, the (a1) gives $c=2 h+1$, and $b=1$, while (b1) gives $d=2 h+1$ and $b=0$, and hence the system has no integer solutions. Assume (a1) and (b2) hold. By (a1) we get, as before, that $c=2 h+1$, and $b=1$, while (b2) gives $d=2 h+1$, and $b=2^{t-1}$, whence again the system is incompatible.

4. We have that $\operatorname{Int}\left(\rho_{1,1}\left(x^{a} y^{2 b}\right)\left(\mathcal{F}_{1,1}\right)\right) \cap \operatorname{Int}\left(\mathcal{F}_{1,1}\right) \cap \Sigma_{1}$ can be non-empty only if one of the following two conditions is fulfilled:

(a1) $2^{t-1} a+(2 h+1) b=2^{t-1}(2 h+1)+c, c=1,2, \ldots, 2^{t-2}-1$,

(a2) $2^{t-1} a+(2 h+1) b=2^{t-1}(2 h+1)-c, c=1,2, \ldots, 2^{t-2}-1$,

while $\operatorname{Int}\left(\rho_{1,1}\left(x^{a} y^{2 b}\right)\left(\mathcal{F}_{1,1}\right)\right) \cap \operatorname{Int}\left(\mathcal{F}_{1,1}\right) \cap \Sigma_{2}$ can be non-empty only if one of the following three conditions is fulfilled:

(b1) $-2^{t-1} a+(2 h+1) b=-d, d=1,2, \ldots, 2 h$,

(b2) $-2^{t-1} a+(2 h+1) b=d, d=1,2, \ldots, 2 h$,

(b3) $-2^{t-1} a+(2 h+1) b=2^{t-1}(2 h+1)-d, d=1,2, \ldots, 2 h$.

Next we show that none of the combinations $(a j), j=1,2$, and $(b k), k=1,2,3$, is possible. Assume, for example, that (a1) and (b1) hold. Then, there are integers a and $b$ such that

$$
\left\{\begin{array}{l}
2^{t-1} a+(2 h+1) b=2^{t-1}(2 h+1)+c \\
-2^{t-1} a+(2 h+1) b=-d
\end{array}\right.
$$

This gives

$$
\left\{\begin{array}{l}
a=h+\frac{1}{2}+\frac{c+d}{2^{t}}, \\
b=\frac{2^{t-1}(2 h+1)+c-d}{2(2 h+1)} .
\end{array}\right.
$$

We show that either $a$ or $b$ is not an integer. If $d=c$, then $\frac{2 c}{2^{t}} \leqslant \frac{2^{t-2}-1}{2^{t-1}}<\frac{1}{2}$, and hence $h<a=h+\frac{1}{2}+\frac{2 c}{2^{t}}<h+1$, and $a$ is not an integer. Next, assume $c \neq d$ and $h \geqslant 2^{t-3}$. Then, since $\min (d)=1$ and $\max (c)=2^{t-2}-1$,

$$
\frac{c-d}{2(2 h+1)} \leqslant \frac{2^{t-3}-1}{2 h+1}<1
$$

While since $\max (d)=2 h$ and $\min (c)=1$,

$$
\frac{c-d}{2(2 h+1)} \geqslant \frac{1-2 h}{2 h+1}>-1 .
$$

Since $c \neq d$, it follows that $b$ is not an integer. The last possibility is $c \neq d$ and $h<2^{t-3}$. Then, $\frac{c}{2^{t}} \leqslant \frac{2^{t-2}-1}{2^{t}}<\frac{1}{4}$ and $\frac{d}{2^{t}} \leqslant \frac{2 h}{2^{t}}<\frac{1}{4}$. This means that: $h<a=h+$ $+\frac{1}{2}+\frac{c+d}{2^{t}}<h+1$, so $a$ is not an integer. The proofs for the other combinations are similar.

5. We find that $\operatorname{Int}\left(\rho_{1,1}\left(x^{a} y^{2 b-1}\right)\left(\mathcal{F}_{1,1}\right)\right) \cap \operatorname{Int}\left(\mathcal{F}_{1,1}\right) \cap \Sigma_{1}$ can be non-empty only if one of the following two conditions is fulfilled:

(a1) $2^{t-1} a+(2 h+1) b=c, c=1,2, \ldots, 2 h+2$,

(a2) $2^{t-1} a+(2 h+1) b=2^{t-1}(2 h+1)-c, c=1,2, \ldots, 2 h+2$, 
while $\operatorname{Int}\left(\rho_{1,1}\left(x^{a} y^{2 b}\right)\left(\mathcal{F}_{1,1}\right)\right) \cap \operatorname{Int}\left(\mathcal{F}_{1,1}\right) \cap \Sigma_{2}$ can be non-empty only if one of the following two conditions is fulfilled:

(b1) $-2^{t-1} a+(2 h+1)(b-1)=-d, d=1,2, \ldots, 2 h+2$,

(b2) $-2^{t-1} a+(2 h+1)(b-1)=2^{t-1}(2 h+1)-d, d=1,2, \ldots, 2 h+2$.

The proof consists of showing that none of the combinations $(a j), j=1,2$, and $(b k), k=1,2$, is possible. Assume, for example, that (a1) and (b1) hold. Then there are integers $a$ and $b$ such that

$$
\left\{\begin{array}{l}
2^{t-1} a+(2 h+1) b=c, \\
-2^{t-1} a+(2 h+1)(b-1)=-d .
\end{array}\right.
$$

This gives

$$
\left\{\begin{array}{l}
a=\frac{-(2 h+1)+c+d}{2^{t}}, \\
b=\frac{1}{2}+\frac{c-d}{2(2 h+1)} .
\end{array}\right.
$$

Next we show that either $a$ or $b$ is not an integer. If $d=c$, then $b=\frac{1}{2}$ is not an integer. Now, assume $c \neq d$. Then, since $\min (d)=1$ and $\max (c)=2 h+2$,

$$
\frac{c-d}{2(2 h+1)} \leqslant \frac{1}{2} .
$$

While since $\max (d)=2 h+2$ and $\min (c)=1$,

$$
\frac{c-d}{2(2 h+1)} \geqslant-\frac{1}{2} \text {. }
$$

So $b=1$ and $c=\frac{1}{2^{t-1}}$. It follows that $c$ is not an integer. The proofs for the other combinations are similar.

The proof of Proposition 3.2 follows by these lemmas and by the fact that $\mathcal{F}_{1,1}$ has the correct volume. Next, we give the fundamental domain $\mathcal{F}_{1, l}$ for the other representations. A pictorial description of $\mathcal{F}_{1, l}$ is given in Figures 5, 6 and 7, which corresponds to Figures 2, 3 and 4 , where $z=m(2 h+1)$, with $l=2 m+1$.

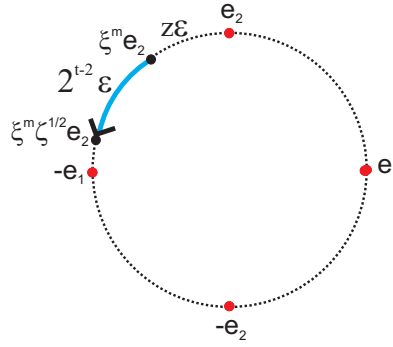

The $\operatorname{arc} R\left(m \theta_{2}\right) A_{1}$

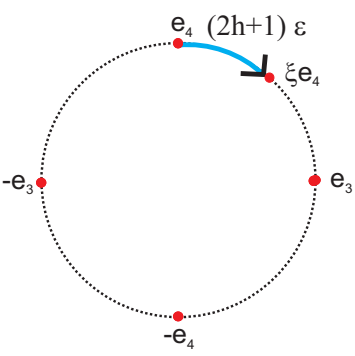

The $\operatorname{arc} \mathrm{A}_{2}$

Figure 5 

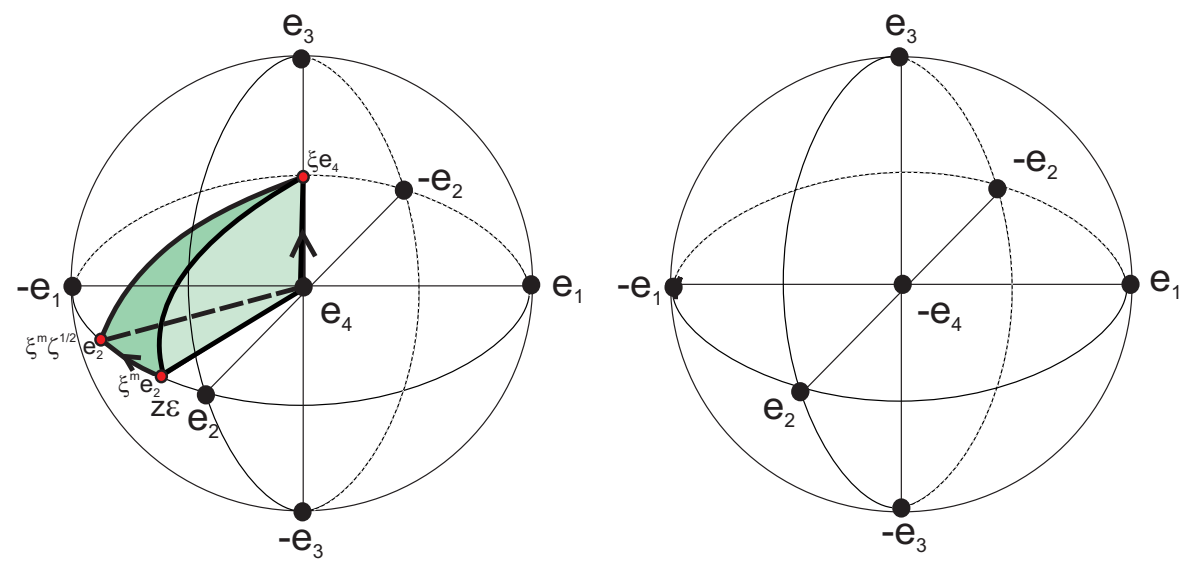

Figure 6

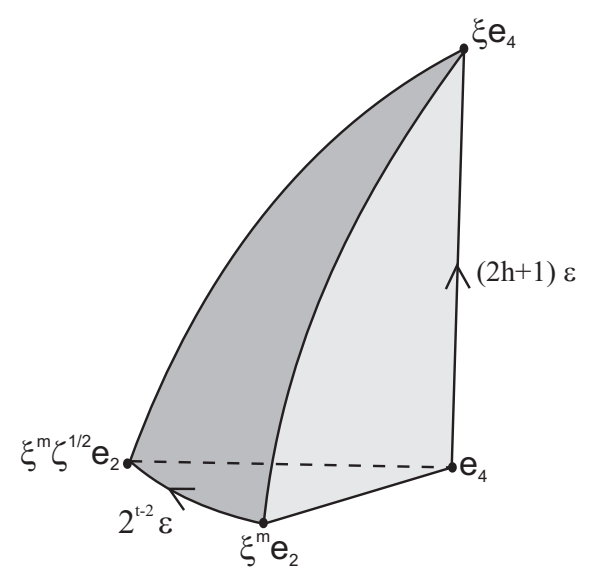

Figure 7

Proposition 3.6. A fundamental domain for the action of the group $D_{2^{t}(2 h+1)}$ on $S^{3}$ via the representation $\pi_{1, l}$, where $l=2 m+1$, is

$$
\mathcal{F}_{1, l}=R\left(m \theta_{2}\right)\left(A_{1}\right) * A_{2}=\left[\xi^{m} i, \xi^{m} \zeta^{\frac{1}{2}} i\right] *[i, \xi i] .
$$

Proof. First, note that the action of the group elements in the representation $\pi_{1, l}$, $l=2 m+1$ is as follows: $\rho_{1, l}(x)=\rho_{1,1}(x)$, while

$$
\rho_{1, l}(y)\left(\alpha_{1} * \alpha_{2}\right)=R\left(l \theta_{2}\right)\left(\alpha_{2}\right) * \alpha_{1},
$$

where $\alpha_{j}$ are arcs in some $\Sigma$, i.e.,

1. $y$ moves $\alpha_{1}$ from $\Sigma_{1}$ into $\Sigma_{2}$,

2. $y$ moves $\alpha_{2}$ from $\Sigma_{2}$ into $\Sigma_{1}$ and rotates it by $(2 h+1) l \varepsilon$ in the positive sense in $\Sigma_{1}$. 
Thus,

$$
\rho_{1, l}(y)\left(\mathcal{F}_{1, l}\right)=R\left(l \theta_{2}\right)\left(A_{2}\right) * R\left(m \theta_{2}\right)\left(A_{1}\right),
$$

and by Remark 3.1,

$$
\begin{aligned}
\rho_{1, l}\left(y^{2 c}\right)\left(\mathcal{F}_{1, l}\right) & =R\left((c l+m) \theta_{2}\right)\left(A_{1}\right) * R\left(c l \theta_{2}\right)\left(A_{2}\right), \\
\rho_{1, l}\left(y^{2 c+1}\right)\left(\mathcal{F}_{1, l}\right) & =R\left((c+1) l \theta_{2}\right)\left(A_{2}\right) * R\left((c l+m) \theta_{2}\right)\left(A_{1}\right) .
\end{aligned}
$$

Second, note that, by definition,

$$
\begin{aligned}
& R\left((c l+m) \theta_{2}\right)\left(A_{1}\right) * R\left((c l+m) \theta_{2}\right)\left(A_{2}\right)=\rho_{1,1}\left(y^{2 c l+2 m}\right)\left(\mathcal{F}_{1,1}\right), \\
& R\left((c+1) l \theta_{2}\right)\left(A_{2}\right) * R\left((c l+2 m) \theta_{2}\right)\left(A_{1}\right)=\rho_{1,1}\left(y^{(2 c+1) l+2 m}\right)\left(\mathcal{F}_{1,1}\right),
\end{aligned}
$$

and

$$
\begin{aligned}
R\left(\operatorname{cl} \theta_{2}\right)\left(A_{1}\right) * R\left(c l \theta_{2}\right) A_{2} & =\rho_{1,1}\left(y^{2 c l}\right)\left(\mathcal{F}_{1,1}\right), \\
R\left((c l+m+1) \theta_{2}\right)\left(A_{2}\right) * R\left((c l+m) \theta_{2}\right)\left(A_{1}\right) & =\rho_{1,1}\left(y^{(2 c+1) l}\right)\left(\mathcal{F}_{1,1}\right) .
\end{aligned}
$$

These equalities show that

$$
\begin{aligned}
& \rho_{1, l}\left(y^{b}\right)\left(\mathcal{F}_{1, l}\right) \cap \Sigma_{1}=\rho_{1,1}\left(y^{b l+2 m}\right)\left(\mathcal{F}_{1,1}\right) \cap \Sigma_{1}, \\
& \rho_{1, l}\left(y^{b}\right)\left(\mathcal{F}_{1, l}\right) \cap \Sigma_{2}=\rho_{1,1}\left(y^{b l}\right)\left(\mathcal{F}_{1,1}\right) \cap \Sigma_{2} .
\end{aligned}
$$

We are now able to prove that the translated of $\mathcal{F}_{1, l}$ do not have common internal points. Assume that

$$
\operatorname{Int}\left(\rho_{1, l}\left(x^{a} y^{b}\right)\left(\mathcal{F}_{1, l}\right) \cap \operatorname{Int}\left(\mathcal{F}_{1, l}\right) \neq \emptyset .\right.
$$

Recalling Lemma 3.3, this means that both affirmations below are true:

$$
\begin{aligned}
& \operatorname{Int}\left(\rho_{1, l}\left(x^{a} y^{b}\right)\left(\mathcal{F}_{1, l}\right)\right) \cap \operatorname{Int}\left(\mathcal{F}_{1, l}\right) \cap \Sigma_{1} \neq \emptyset, \\
& \operatorname{Int}\left(\rho_{1, l}\left(x^{a} y^{b}\right)\left(\mathcal{F}_{1, l}\right)\right) \cap \operatorname{Int}\left(\mathcal{F}_{1, l}\right) \cap \Sigma_{2} \neq \emptyset .
\end{aligned}
$$

Suppose first that

$$
\operatorname{Int}\left(\rho_{1, l}\left(x^{a} y^{b}\right)\left(\mathcal{F}_{1, l}\right)\right) \cap \operatorname{Int}\left(\mathcal{F}_{1, l}\right) \cap \Sigma_{1} \neq \emptyset .
$$

By equation (3.1), this means that

$$
\operatorname{Int}\left(\rho_{1,1}\left(x^{a} y^{b l+2 m}\right)\left(\mathcal{F}_{1,1}\right)\right) \cap \operatorname{Int}\left(\mathcal{F}_{1, l}\right) \cap \Sigma_{1} \neq \emptyset,
$$

and by definition this gives the equation (in $\Sigma_{1}$ )

$$
\begin{cases}R\left(a \theta_{1}+(c l+m) \theta_{2}\right)\left(\operatorname{Int}\left(A_{1}\right)\right) \cap R\left(a \theta_{1}+m \theta_{2}\right)\left(\operatorname{Int}\left(A_{1}\right)\right) \neq \emptyset, & b=2 c, \\ R\left(a \theta_{1}+(c+1) l \theta_{2}\right)\left(\operatorname{Int}\left(A_{2}\right)\right) \cap R\left(a \theta_{1}+m \theta_{2}\right)\left(\operatorname{Int}\left(A_{1}\right)\right) \neq \emptyset, & b=2 c+1 .\end{cases}
$$

Since this is just an equation in $\Sigma_{1}$, it is invariant under rotation, whence equivalent to the equation

$$
\begin{cases}R\left(a \theta_{1}+c l \theta_{2}\right)\left(\operatorname{Int}\left(A_{1}\right)\right) \cap \operatorname{Int}\left(A_{1}\right) \neq \emptyset, & b=2 c, \\ R\left(a \theta_{1}+(c l+m+1) \theta_{2}\right)\left(\operatorname{Int}\left(A_{2}\right)\right) \cap \operatorname{Int}\left(A_{1}\right) \neq \emptyset, & b=2 c+1 .\end{cases}
$$

By definition, equation (3.4) comes from the condition

$$
\operatorname{Int}\left(\rho_{1,1}\left(x^{a} y^{b l}\right)\left(\mathcal{F}_{1,1}\right)\right) \cap \operatorname{Int}\left(\mathcal{F}_{1,1}\right) \cap \Sigma_{1} \neq \emptyset .
$$


Since $\mathcal{F}_{1,1}$ is a fundamental domain with respect to the representation $\rho_{1,1}$, condition (3.5) implies that

$$
\operatorname{Int}\left(\rho_{1,1}\left(x^{a} y^{b l}\right)\left(\mathcal{F}_{1,1}\right)\right) \cap \operatorname{Int}\left(\mathcal{F}_{1,1}\right) \cap \Sigma_{2}=\emptyset,
$$

and by (3.2), and recalling that $\mathcal{F}_{1, l} \cap \Sigma_{2}=\mathcal{F}_{1,1} \cap \Sigma_{2}$, we have that

$$
\operatorname{Int}\left(\rho_{1, l}\left(x^{a} y^{b l}\right)\left(\mathcal{F}_{1, l}\right)\right) \cap \operatorname{Int}\left(\mathcal{F}_{1, l}\right) \cap \Sigma_{2}=\emptyset .
$$

So we have proved that condition (3.3) implies condition (3.6). Next, observe that all the implications used to prove the last equation are in fact double implications, and hence condition (3.6) implies condition (3.3). This conclude the proof.

\subsection{Cellular decomposition}

In whole generality, if $X=S^{k} / \pi$, where $\pi$ is some finite group acting freely by isometries, $\mathcal{F}$ is the fundamental domain of the action of $\pi$, and $q: S^{n} \rightarrow X$ is the natural quotient map, then $q$ is a covering map, $S^{n}=\tilde{X}$ is the universal covering space of $X,\left.q\right|_{\mathcal{F}}: \mathcal{F} \rightarrow X$ is surjective, and it is a relative homomorphism for the pair $(\mathcal{F}, \partial \mathcal{F}) \rightarrow \operatorname{Int}(\operatorname{Top}(\mathrm{X}))$, onto the interior of the top cell of $X$ (recall $X$ is a compact manifold). It is clear that a $\pi$ equivariant cell decomposition $\tilde{K}$ of $S^{n}$ determines a cell decomposition $\tilde{L}$ of $\mathcal{F}$, where $\tilde{L}$ is a subcomplex of $\tilde{K}$, and $\tilde{K}=\pi \tilde{L}$, and that $q(\tilde{L})$ will give a cell decomposition $L$ of $X$. Moreover, at least one lift of each cell of $L$ will lay in $\tilde{L}$. Therefore, we can choose for each cell $c \in L$ one single cell $\tilde{c} \in \tilde{L}$, which will be called a representative lift of $c$. Since all the other cells of $\tilde{L}$ are in the $\pi$ orbit of some cell $\tilde{c}$ (i.e., are translated by the action of some group element), the cell complex of $\tilde{L}$ can be described using the cells $\tilde{c}$ and some of their translations. This set of cells $\tilde{Z}$ will be called a minimal set of representative lifts, and $\tilde{Z}$ a minimal cell decomposition of $\mathcal{F}$. It is clear that $q(\tilde{Z})=L$. Taking all the complete orbits of the cells in $\tilde{Z}$, we will obtain the cell complex $\tilde{K}=\pi \tilde{Z}$, which is a $\pi$ equivariant cellular decomposition of $S^{n}$.

We pass now to determine a $D_{2^{t}(2 h+1)}$ equivariant cell decomposition of $S^{3}$. By Proposition 3.6, $\mathcal{F}_{1, l}=A_{1}(l) * A_{2} \subseteq \Sigma_{1} * \Sigma_{2}$, where $A_{1}(l)=R\left(m \theta_{2}\right) A_{1}$, as defined in the theorem, and for simplicity will be denoted by $A_{1}$ here. The $A_{j}$ are segments of geodesic arcs in the two factors $\Sigma_{j}$ of $S^{3}=S^{1} * S^{1}$. The end points of the $A_{j}$ are obviously images of some common point of $S^{3}$ by the action of some different group elements. This defines a cell decomposition $\tilde{N}_{j}$ of the $\Sigma_{j}$, as follows: Fix a point of $S^{3}$, and identify this point with $e_{4}$. The 0 -cells of $\tilde{N}_{j}$ are the elements of the orbit $D_{2^{t}(2 h+1)} e_{4}$ that lay in $\Sigma_{j}$. The 1-cells of $\tilde{N}_{j}$ are the geodesic arc segments joining such points (that all lay in $\Sigma_{j}$, since the action is orthogonal). It is clear that the $A_{j}$ are realized by two subcomplexes $B_{j} \subseteq N_{j}$. Since the join preserves subcomplexes, the join $\tilde{L}=\tilde{B}_{1} * \tilde{B}_{2}$ gives a cell decomposition of $\mathcal{F}_{1, l}$. It is clear that $\tilde{K}=D_{2^{t}(2 h+1)} \tilde{L}$ is a $D_{2^{t}(2 h+1)}$ equivariant cell decomposition of $S^{3}=\Sigma_{1} * \Sigma_{2}$. This gives the following lemma:

Lemma 3.7. $A D_{2^{t}(2 h+1)}$ equivariant cell decomposition of $S^{3}$ is $\tilde{K}=D_{2^{t}(2 h+1)} \tilde{L}$, where $\tilde{L}$ is defined above. The subcomplex $\tilde{L}$ is a cell decomposition of $\mathcal{F}_{1, l}$. The quotient $L=\tilde{L} / \pi_{k, l}\left(D_{2^{t}(2 h+1)}\right)$ gives a cell decomposition of $\mathbf{S}_{2^{t}(2 h+1) ; k ; l}^{3}$.

In order to proceed, we need the group elements that define the minimal action on each of the two circles $\Sigma_{j}$, i.e., the two elements $g_{j} \in D_{2^{t}(2 h+1)}$ such that, for any 
given fixed $z_{j} \in \Sigma_{j}, j=1,2$,

$$
g_{j} z_{j} * z_{j}=\epsilon
$$

A straightforward calculation gives the following relationships:

Lemma 3.8. The elements $g_{j} \in D_{2^{t}(2 h+1)}$, which satisfy equation (3.7), are $g_{j}=$ $x^{\alpha_{j}} y^{2 \beta_{j}}$, where $\alpha_{j}$ and $\beta_{j}$ are integers satisfying the following equations:

$$
\begin{aligned}
& k 2^{t-1} \alpha_{1}+l(2 h+1) \beta_{1}=1 \quad \bmod 2^{t-1}(2 h+1), \\
& -k 2^{t-1} \alpha_{2}+l(2 h+1) \beta_{2}=-1 \quad \bmod 2^{t-1}(2 h+1) \text {. }
\end{aligned}
$$

It is easy to see that if the pair $\left(\alpha_{1}, \beta_{1}\right)$ satisfies the first equation of Lemma 3.8, then the pair $\left(\alpha_{2}=\alpha_{1}, \beta_{2}=-\beta_{1}\right)$ satisfies the second equation of the lemma. Thus, we define the pair $(\alpha, \beta)$ as the two integers that satisfy the following equation:

$$
k 2^{t-1} \alpha+l(2 h+1) \beta=1 \bmod 2^{t-1}(2 h+1) .
$$

We are now in the position of describing the minimal set of lifts $\tilde{Z}$ of the cells of $L$ in $S^{3}$ and their boundaries. In order to describe this complex, we use a "semialgebraic" notation, namely, after fixing one 0 -cell, $e_{4}$, we denote the other 0-cells by action of the groups elements. Higher dimensional cells are obtained by joining lower dimensional ones as before. Addition is used to denote cells constructed by glueing adjacent cells.

Since the description changes if $2 h+1$ is greater or smaller than $2^{t-2}$, we split the statement in the following two lemmas. The proof is the same.

Proposition 3.9. Let $z=m(2 h+1)$, and let $(\alpha, \beta)$ be integers satisfying the conditions given in equation (3.8). Assume $2 h+1>2^{t-2}$. Then, a minimal set $\tilde{Z}$ of representative lifts $\tilde{c}_{q, s}$ (where the first index denotes the dimension) of the cells of the cellular decomposition $L$ of $\mathbf{S}_{2^{t}(2 h+1) ; k ; l}^{3}$ (recall $\left.l=2 m+1\right)$ defined in Lemma 3.7 is:

$$
\begin{aligned}
& \tilde{c}_{0,1}=e_{4}, \\
& \tilde{c}_{1,1}=e_{4} *\left(x^{\alpha} y^{-2 \beta}\right) e_{4}, \\
& \tilde{c}_{1,2}=e_{4} *\left(x^{\alpha} y^{2 \beta}\right)^{z+2^{t-2}} y^{-1} e_{4}, \\
& \tilde{c}_{1,3}=e_{4} *\left(x^{\alpha} y^{2 \beta}\right)^{z} y^{-1} e_{4}, \\
& \tilde{c}_{2,1}=\left(x^{\alpha} y^{2 \beta}\right)^{z} y^{-1} e_{4} *\left(x^{\alpha} y^{-2 \beta}\right)^{2 h+1} e_{4} *\left(x^{\alpha} y^{2 \beta}\right)^{z+2^{t-2}} y^{-1} e_{4}, \\
& \tilde{c}_{2,2}=\left(x^{\alpha} y^{-2 \beta}\right)^{2 h+1-2^{t-2}} e_{4} *\left(x^{\alpha} y^{-2 \beta}\right)^{2 h+1} e_{4} *\left(x^{\alpha} y^{2 \beta}\right)^{z} y^{-1} e_{4}, \\
& \tilde{c}_{2,3}=e_{4} *\left(x^{\alpha} y^{-2 \beta}\right)^{2 h+1-2^{t-2}} e_{4} *\left(x^{\alpha} y^{2 \beta}\right)^{z} y^{-1} e_{4}, \\
& \tilde{c}_{3,1}=\sum_{s=0}^{2 h}\left(x^{\alpha} y^{-2 \beta}\right)^{s} \tilde{c}_{1,1} * \sum_{s=1}^{2^{t-2}}\left(x^{\alpha} y^{2 \beta}\right)^{z+s} y^{-1} \tilde{c}_{1,1},
\end{aligned}
$$

with the boundaries: 


$$
\begin{aligned}
\partial_{1}\left(\tilde{c}_{1,1}\right)= & \left(x^{\alpha} y^{-2 \beta}-1\right) \tilde{c}_{0,1}, \\
\partial_{1}\left(\tilde{c}_{1,2}\right)= & \left(\left(x^{\alpha} y^{2 \beta}\right)^{z+2^{t-2}} y^{-1}-1\right) \tilde{c}_{0,1}, \\
\partial_{1}\left(\tilde{c}_{1,3}\right)= & \left(\left(x^{\alpha} y^{2 \beta}\right)^{z} y^{-1}-1\right) \tilde{c}_{0,1}, \\
\partial_{2}\left(\tilde{c}_{2,1}\right)= & \sum_{s=1}^{2^{t-2}}\left(x^{\alpha} y^{2 \beta}\right)^{z+s} y^{-1} \tilde{c}_{1,1}-\left(x^{\alpha} y^{2 \beta}\right)^{z+2^{t-2}} y^{-1} \tilde{c}_{1,2}+\left(x^{\alpha} y^{2 \beta}\right)^{z} y^{-1} \tilde{c}_{1,3}, \\
\partial_{2}\left(\tilde{c}_{2,2}\right)= & \sum_{s=2 h+1-2^{t-2}}^{2 h}\left(x^{\alpha} y^{-2 \beta}\right)^{s} \tilde{c}_{1,1}+\left(x^{\alpha} y^{2 \beta}\right)^{z} y^{-1} \tilde{c}_{1,2}-\left(x^{\alpha} y^{2 \beta}\right)^{z} y^{-1} \tilde{c}_{1,3}, \\
\partial_{2}\left(\tilde{c}_{2,3}\right)= & \sum_{s=0}^{2 h-2^{t-2}}\left(x^{\alpha} y^{-2 \beta}\right)^{s} \tilde{c}_{1,1}-\left(x^{\alpha} y^{2 \beta}\right)^{z} y^{-1} \tilde{c}_{1,2}-\tilde{c}_{1,3}, \\
\partial_{3}\left(\tilde{c}_{3,1}\right)= & \left(1-\left(\left(x^{\alpha} y^{2 \beta}\right)^{z+2^{t-2}} y^{-1}\right)^{-1}\right) \tilde{c}_{2,1}+\left(1-\left(\left(x^{\alpha} y^{2 \beta}\right)^{z} y^{-1}\right)^{-1}\right) \tilde{c}_{2,2} \\
& +\left(1-\left(x^{\alpha} y^{-2 \beta}\right)^{2^{t-2}}\right) \tilde{c}_{2,3} .
\end{aligned}
$$

Proof. The proof of this lemma is geometric, and it is based on an explicit description of the cell decomposition $\tilde{L}$ of the fundamental domain. First, since $\mathbf{S}_{2^{t}(2 h+1) ; \mathbf{k} ; \mathbf{l}}^{3}$ is connected, one 0 -cell $c_{0,0}$ is sufficient in $L$, and we fix the lift $\tilde{c}_{0,0}$ of $c_{0,0}$ by identifying it with the end point of the basis vector $e_{4}$. Next, since the quotient space is a manifold and the fundamental domain is a 3-dimensional disc, it is clear that we can take just one top cell. Namely, we can lift the top cell of $L$, and this will be exactly $\tilde{L}$, with the boundary glued on the 2-skeleton of $\tilde{L}$, which is precisely its boundary. By definition, $\tilde{L}=\tilde{B}_{1} * \tilde{B}_{2}$. So the next step is to identify the $\tilde{B}_{j}$. The main problem here is that it is not difficult to realize that the two 1-dimensional complexes $\tilde{B}_{j}$ are, in fact, in the same $D_{2^{t}(2 h+1)}$ orbit, and hence they cannot give a set of lifts of 1-cells. This can be seen in detail in the content of Lemma 3.8. In fact, by definition, the 0 -cells of $\tilde{B}_{j}$ are the points in the orbit of $e_{4}$ that belong to $A_{j}$. By definition (Proposition 3.6), $A_{1}(l)=\left(x^{\alpha_{1}} y^{2 \beta_{1}}\right)^{z} e_{2} *$ $\left(x^{\alpha_{1}} y^{2 \beta_{1}}\right)^{z+2^{t-2}} e_{2}=\left(x^{\alpha_{1}} y^{2 \beta_{1}}\right)^{z} y^{2^{t}-1} e_{4} *\left(x^{\alpha_{1}} y^{2 \beta_{1}}\right)^{z+2^{t-2}} y^{2^{t}-1} e_{4}$, since $e_{2}=y^{2^{t}-1} e_{4}$, and $A_{2}=e_{4} *\left(x^{\alpha_{2}} y^{2 \beta_{2}}\right)^{2 h+1} e_{4}\left(\left(\alpha_{j}, \beta_{j}\right)\right.$ are defined in Lemma 3.8). Thus, the points of the orbit of $e_{4}$ that belong to $\tilde{B}_{1}$ are the $g_{1}^{u} y^{2^{t}-1} e_{4}$, where $g_{1}=x^{\alpha_{1}} y^{2 \beta_{1}}$, and $z \leqslant u \leqslant z+2^{t-2}$, while the points of the orbit of $e_{4}$ that belong to $\tilde{B}_{2}$ are the $g_{2}^{u} e_{4}$, where $g_{2}=x^{\alpha_{2}} y^{2 \beta_{2}}$, and $0 \leqslant u \leqslant 2 h+1$. Thus, choosing one 1-cell

$$
\tilde{c}_{1,1}=e_{4} *\left(x^{\alpha_{2}} y^{2 \beta_{2}}\right) e_{4},
$$

we have that

$$
\tilde{B}_{1}=\bigcup_{u=z}^{z+2^{t-2}}\left(x^{\alpha_{1}} y^{2 \beta_{1}}\right)^{u} y^{2^{t}-1} \tilde{c}_{1,1}, \quad \tilde{B}_{2}=\bigcup_{u=0}^{2 h}\left(x^{\alpha_{2}} y^{2 \beta_{2}}\right)^{u} \tilde{c}_{1,1} .
$$

We are now in the position of giving representative lifts in $\tilde{Z}$ of the 1-cells of $L$. Note that the 1 skeleton of $\tilde{L}$ contains, beside the $\tilde{B}_{j}$, the $\operatorname{arcs}$ segments $I_{1}=e_{4} *$ $\left(x^{\alpha_{1}} y^{2 \beta_{1}}\right)^{z+2^{t-2}} y^{2^{t}-1} e_{4}, \quad I_{2}=e_{4} *\left(x^{\alpha_{1}} y^{2 \beta_{1}}\right)^{z} y^{2^{t}-1} e_{4}, \quad I_{1}^{\prime}=\left(x^{\alpha_{1}} y^{2 \beta_{1}}\right)^{z+2^{t-2}} y^{2^{t}-1} e_{4} *$ $\left(x^{\alpha_{2}} y^{2 \beta_{2}}\right)^{2 h+1} e_{4}$ and $I_{2}^{\prime}=\left(x^{\alpha_{1}} y^{2 \beta_{1}}\right)^{z} y^{2^{t}-1} e_{4} *\left(x^{\alpha_{2}} y^{2 \beta_{2}}\right)^{2 h+1} e_{4}$; see Figure 8. (The 
corresponding figure for the case $2 h+1<2^{t-2}$ is Figure 9.) We see that $I_{1}^{\prime}$ and $I_{1}$ and $I_{2}^{\prime}$ and $I_{2}$ are respectively in the same orbit, and none of them is in some orbit of the $N_{j}$. Thus we choose the minimal set of lifts of 1-cells to be the set:

$$
\begin{aligned}
& \tilde{c}_{1,1}=e_{4} *\left(x^{\alpha_{2}} y^{2 \beta_{2}}\right) e_{4}, \\
& \tilde{c}_{1,2}=e_{4} *\left(x^{\alpha_{1}} y^{2 \beta_{1}}\right)^{z+2^{t-2}} y^{2^{t}-1} e_{4}, \\
& \tilde{c}_{1,3}=e_{4} *\left(x^{\alpha_{1}} y^{2 \beta_{1}}\right)^{z} y^{2^{t}-1} e_{4},
\end{aligned}
$$

where the last two cells represent, respectively, onto $I_{1}$ and $I_{2}$.

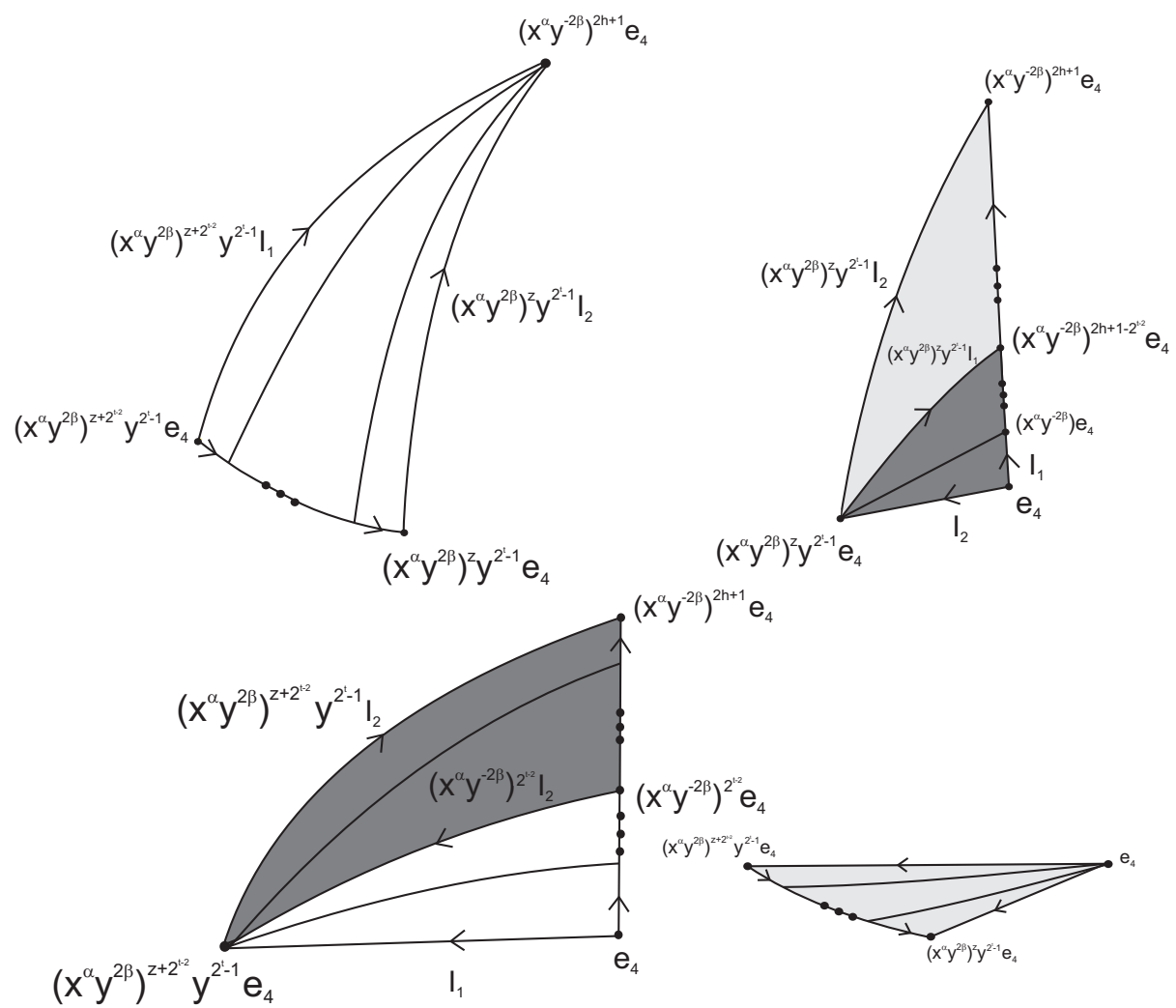

Figure 8

We pass now to choose the 2-cells of $\tilde{Z}$. The procedure is similar; we need to identify the orbits of the two cells in $\tilde{L}$. Figure 8 can again be useful, where the three 2 -cells with different orbits are displayed in different colors. The final minimal set of 2-cells is given in the statement. It is not difficult to see that the unique 3-cell of $\tilde{Z}$ is $\tilde{c}_{3_{1}}$, and is, in fact, the whole $\tilde{L}$.

Eventually, we compute the boundaries using Lemma 2.5, and we write the coefficients in $\mathbb{Z} D_{2^{t}(2 h+1)}$ using the known description of the orbit of the point $e_{4}$. This completes the proof. 

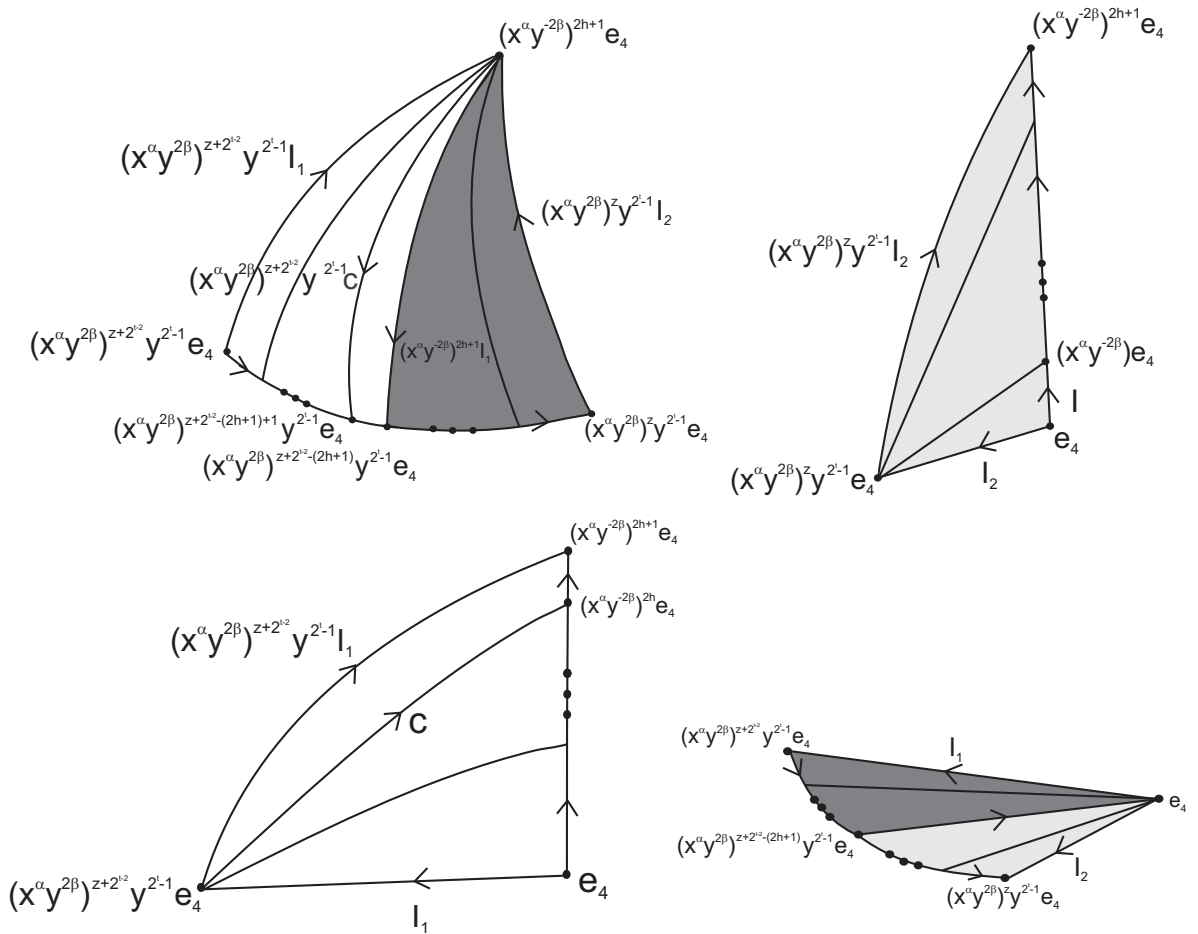

Figure 9

Proposition 3.10. Let $z=m(2 h+1)$, and let $(\alpha, \beta)$ be integers satisfying the conditions given in equation (3.8). Assume $2 h+1<2^{t-2}$. Then, a minimal set $\tilde{Z}$ of representative lifts $\tilde{c}_{q, s}$ (where the first index denotes the dimension) of the cells of the cellular decomposition $L$ of $\mathbf{S}_{2^{t}(2 h+1) ; k ; l}^{3}$ (recall $l=2 m+1$ ) defined in Lemma 3.7 is:

$$
\begin{aligned}
& \tilde{c}_{0,1}=e_{4}, \\
& \tilde{c}_{1,1}=e_{4} *\left(x^{\alpha} y^{-2 \beta}\right) e_{4}, \\
& \tilde{c}_{1,2}=e_{4} *\left(x^{\alpha} y^{2 \beta}\right)^{z+2^{t-2}} y^{-1} e_{4}, \\
& \tilde{c}_{1,3}=e_{4} *\left(x^{\alpha} y^{2 \beta}\right)^{z} y^{-1} e_{4}, \\
& \tilde{c}_{2,1}=\left(x^{\alpha} y^{2 \beta}\right)^{z+2^{t-2}-(2 h+1)} y^{-1} e_{4} *\left(x^{\alpha} y^{-2 \beta}\right)^{2 h+1} e_{4} *\left(x^{\alpha} y^{2 \beta}\right)^{z+2^{t-2}} y^{-1} e_{4}, \\
& \tilde{c}_{2,2}=\left(x^{\alpha} y^{2 \beta}\right)^{z} y^{-1} e_{4} *\left(x^{\alpha} y^{-2 \beta}\right)^{2 h+1} e_{4} *\left(x^{\alpha} y^{2 \beta}\right)^{z+2^{t-2}-(2 h+1)} y^{-1} e_{4}, \\
& \tilde{c}_{2,3}=e_{4} *\left(x^{\alpha} y^{-2 \beta}\right)^{2 h+1} e_{4} *\left(x^{\alpha} y^{2 \beta}\right)^{z} y^{-1} e_{4}, \\
& \tilde{c}_{3,1}=\sum_{s=0}^{2 h}\left(x^{\alpha} y^{-2 \beta}\right)^{s} \tilde{c}_{1,1} * \sum_{s=1}^{2^{t-2}}\left(x^{\alpha} y^{2 \beta}\right)^{z+s} y^{-1} \tilde{c}_{1,1},
\end{aligned}
$$

with the boundaries: 


$$
\begin{aligned}
\partial_{1}\left(\tilde{c}_{1,1}\right)= & \left(x^{\alpha} y^{-2 \beta}-1\right) \tilde{c}_{0,1} \\
\partial_{1}\left(\tilde{c}_{1,2}\right)= & \left(\left(x^{\alpha} y^{2 \beta}\right)^{z+2^{t-2}} y^{-1}-1\right) \tilde{c}_{0,1} \\
\partial_{1}\left(\tilde{c}_{1,3}\right)= & \left(\left(x^{\alpha} y^{2 \beta}\right)^{z} y^{-1}-1\right) \tilde{c}_{0,1} \\
\partial_{2}\left(\tilde{c}_{2,1}\right)= & \sum_{s=2^{t-2}-2 h}^{2^{t-2}}\left(x^{\alpha} y^{2 \beta}\right)^{z+s} y^{-1} \tilde{c}_{1,1}-\left(\left(x^{\alpha} y^{2 \beta}\right)^{z+2^{t-2}} y^{-1}+\left(x^{\alpha} y^{-2 \beta}\right)^{2 h+1}\right) \tilde{c}_{1,2}, \\
\partial_{2}\left(\tilde{c}_{2,2}\right)= & \sum_{s=1}^{2^{t-2}-(2 h+1)}\left(x^{\alpha} y^{2 \beta}\right)^{z+s} y^{-1} \tilde{c}_{1,1}+\left(x^{\alpha} y^{-2 \beta}\right)^{2 h+1} \tilde{c}_{1,2}+\left(x^{\alpha} y^{2 \beta}\right)^{z} y^{-1} \tilde{c}_{1,3}, \\
\partial_{2}\left(\tilde{c}_{2,3}\right)= & \sum_{s=0}^{2 h}\left(x^{\alpha} y^{-2 \beta}\right)^{s} \tilde{c}_{1,1}-\left(\left(x^{\alpha} y^{2 \beta}\right)^{z} y^{-1}+1\right) \tilde{c}_{1,3}, \\
\partial_{3}\left(\tilde{c}_{3,1}\right)= & \left(1-\left(\left(x^{\alpha} y^{2 \beta}\right)^{z+2^{t-2}} y^{-1}\right)^{-1}\right) \tilde{c}_{2,1}+\left(1-\left(\left(x^{\alpha} y^{-2 \beta}\right)^{2 h+1}\right)^{-1}\right) \tilde{c}_{2,2} \\
& +\left(1-\left(\left(x^{\alpha} y^{2 \beta}\right)^{z} y^{-1}\right)^{-1}\right) \tilde{c}_{2,3} .
\end{aligned}
$$

We displayed in Figures 10 (case $2 h+1>2^{t-2}$ ) and 11 (case $2 h+1<2^{t-2}$ ) the cells of the decomposition $L$ of $\mathbf{S}_{2^{t}(2 h+1) ; k ; l}^{3}$, which are in bijective correspondence with the cells of the minimal set $\tilde{Z}$.

\section{The higher dimensional cases}

The definition of a fundamental domain for the action of the split metacyclic groups on the higher dimensional spheres and the construction of the equivariant cell complex of $S^{4 n-1}$ are based on the following general result, which permits us to reduce the natural cellular decomposition of a join of spheres by considering blocks of cells. Recall the action on the join representation of the spheres, as described at the end of Section 2.4.

Lemma 4.1. Let $\pi$ be a finite group acting freely and orthogonally on a sphere $S^{n}$. Assume we have a $\pi$-cellular decomposition $\tilde{K}$ of $S^{n}$, with fundamental domain $\mathcal{F}$, and hence that we have a cellular decomposition $\tilde{L}$ of $\mathcal{F}$, where $\tilde{L}$ is a subcomplex of $\tilde{K}, \tilde{K}=\pi \tilde{L}$, and $L=\tilde{L} / \pi$ is a cell decomposition of $S^{n} / \pi$. Assume that we have a subcomplex $\tilde{Z}$ of $\tilde{L}$ that is a minimal decomposition of $\mathcal{F}$ of lifts of the cells of $L$. Let $k$ be a positive integer $k \leqslant 2$, and consider the natural action of $\pi$ on $S^{k(n+1)-1}$ in equation (2.2). Then we have a $\pi$-cellular decomposition $\tilde{K}^{\prime}$ of $S^{k(n+1)-1}$, with fundamental domain $\mathcal{F}^{\prime}=S^{(k-1)(n+1)-1} * \mathcal{F}$, and a minimal cell decomposition $\tilde{L}^{\prime}$ of $\mathcal{F}^{\prime}$, where $\tilde{L}^{\prime}$ is defined as follows: The $(k-1)(n+1)-1$ skeleton of $\tilde{L}^{\prime}$ is $\tilde{L}_{((k-1)(n+1)-1)}^{\prime}=\tilde{K}$. The $(k-1)(n+1)$ skeleton $\tilde{L}_{((k-1)(n+1))}^{\prime}$ of $\tilde{L}^{\prime}$ is obtained by attaching $k_{0}(k-1)(n+1)$-cells to $\tilde{L}_{((k-1)(n+1)-1)}^{\prime}=\tilde{K}$, where $k_{0}$ is the number of 0 -cells $\tilde{c}_{0, l}$ of $\tilde{Z}$ and the attaching map is given by the join $\tilde{K} * \tilde{c}_{0, l}$. The $(k-1)(n+1)+1$ skeleton of $\tilde{L}^{\prime}$ is obtained by attaching $k_{1}(k-1)(n+1)+1$-cells to the $(k-1)(n+1)$ skeleton $\tilde{L}_{((k-1)(n+1))}^{\prime}$, where $k_{1}$ is the number of 1-cells $\tilde{c}_{1, l}$ of $\tilde{Z}$ and the attaching map is given by the joint $\tilde{L}_{((k-1)(n+1))}^{\prime} * \tilde{c}_{1, l}$. This procedure 

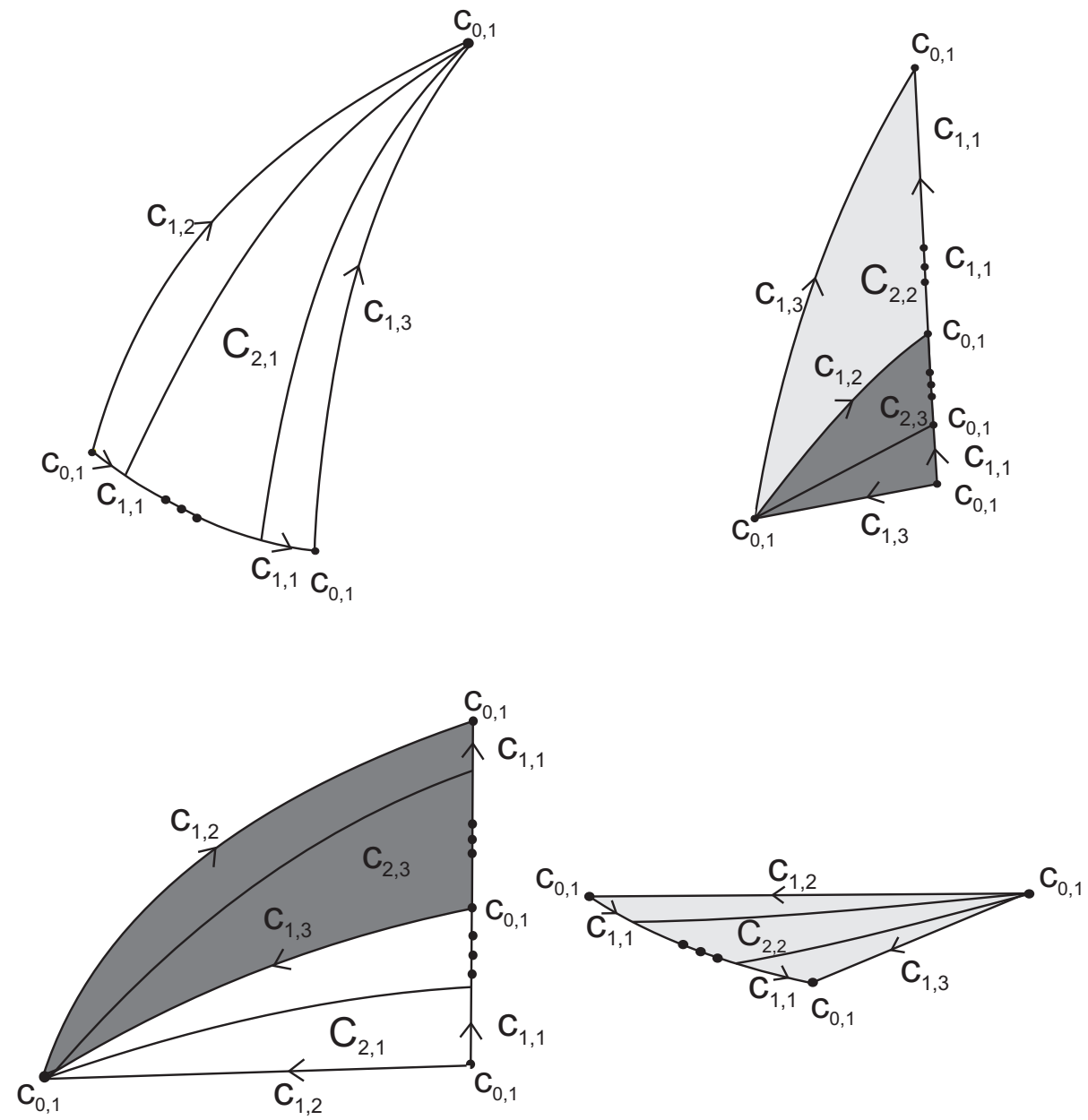

Figure 10

continues up to dimension $k(n+1)-1$, giving the cell decompositions $\tilde{L}^{\prime}$ of $\mathcal{F}^{\prime}$, and $\tilde{K}^{\prime}=\pi \tilde{L}^{\prime}$ of $S^{k(n+1)-1}$.

Proof. Identify $S^{k(n+1)-1}$ with $S^{(k-1)(n+1)-1} * S^{n}$. It is clear by the definition of join, that the join $\tilde{B}=\tilde{K} * \tilde{K}$ provides a $\pi$ cellular decomposition of $S^{(k-1)(n+1)-1} * S^{n}$. Since the join preserves subcomplexes, $\tilde{C}=\tilde{K} * \tilde{L}$ is a cell decomposition of $\mathcal{F}^{\prime}=$ $S^{(k-1)(n+1)-1} * \mathcal{F}$. We now define a new decomposition $\tilde{L}^{\prime}$ of $\mathcal{F}^{\prime}$ reducing the number of the cells of $\tilde{C}$ by defining new cells that are blocks of the cells of $\tilde{B}$. This procedure simplifies the resolution of the group $\mathbb{Z}[\pi]$.

First, let the subcomplex $\tilde{L}_{((k-1)(n+1)-1)}^{\prime}=\tilde{K} * \emptyset=\left\{\left[x, 0, y_{0}\right]\right\}$ of $\tilde{K} * \tilde{L}$ be the $(k-1)(n+1)-1$ skeleton of $\tilde{L}^{\prime}$. Note that $\tilde{C}$ is a $k(n+1)-1$ disc, and that the boundary of $\tilde{C}$ is precisely $\tilde{K} * \partial \tilde{L}$, because $\tilde{K}$ has no boundary. Thus, the action of $\pi$ onto $\tilde{B}$ will send the interior of $\tilde{C}$ in sets that intersect only in their boundaries, 

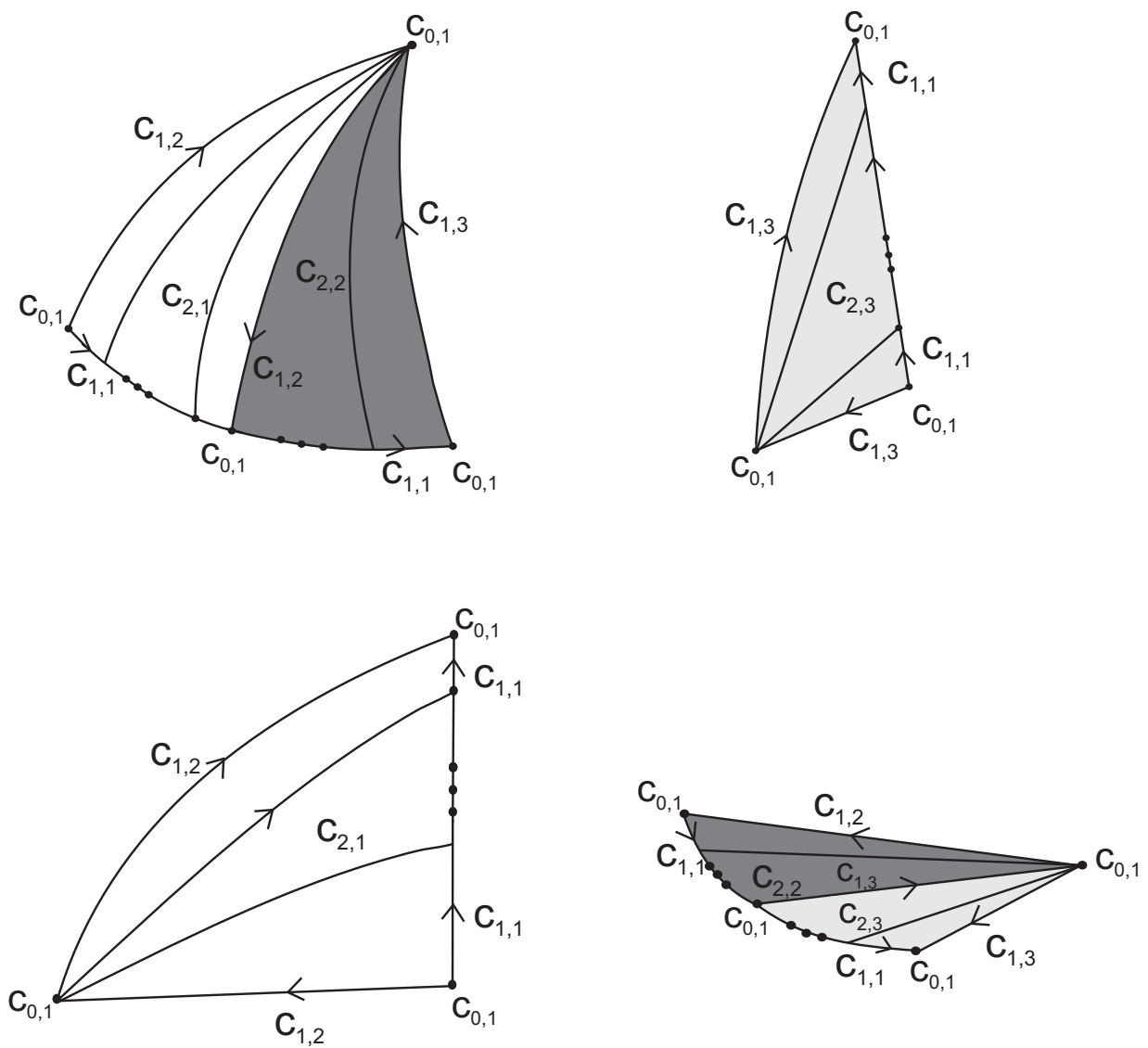

Figure 11

and hence $\tilde{C}$ is a fundamental domain of the $\pi$ action on $\tilde{B}$.

Next, we start the construction of $\tilde{L}^{\prime}$. Let $c_{0, j}$ be a 0 -cell of $L$, and $\tilde{c}_{0, j}$ a lift of $c_{0, j}$ that lays inside $\tilde{L}$. Include $\tilde{c}_{0, j}$ in $\tilde{C}$ as $\emptyset * \tilde{c}_{0, j}$. Then, $\emptyset * \tilde{c}_{0, j}$ belongs to $\tilde{K} *$ $\partial \tilde{L}$, and $\tilde{K} * \tilde{c}_{0, j}$ is a disc of dimension $(k-1)(n+1)$, that is, in fact, a block of cells of dimensions up to $(k-1)(n+1)$ of $\tilde{C}$. Note that, by definition of the join, the interiors of each pair of discs $\tilde{K} * \tilde{c}_{0, j}$ and $\tilde{K} * \tilde{c}_{0, h \neq j}$ are disjoint, and that the intersection of the boundaries lays in $\tilde{K} * \emptyset$. Let the $\operatorname{discs} \tilde{c}_{(k-1)(n+1), j}^{\prime}=\tilde{K} * \tilde{c}_{0, j}$ be the $k_{0}(k-1)(n+1)$ cells of $\tilde{L}^{\prime}$. They are attached to the $(k-1)(n+1)-1$ skeleton by the definition of the join. The $(k-1)(n+1)$ skeleton of $\tilde{L}^{\prime}$ is $\tilde{L}_{((k-1)(n+1))}^{\prime}=$ $\bigcup \tilde{c}_{(k-1)(n+1), j}^{\prime}$.

Let $c_{1, j}$ be a 1-cell of $L$, and $\tilde{c}_{1, j}$ a lift of $c_{1, j}$ that lays inside $\tilde{L}$. Include $\tilde{c}_{1, j}$ in $\tilde{C}$ as $\emptyset * \tilde{c}_{1, j}$. Then, $\emptyset * \tilde{c}_{1, j}$ belongs to $\tilde{K} * \partial \tilde{L}$. Moreover, $\tilde{c}_{(k-1)(n+1)+1, j}^{\prime}=\tilde{K} * \tilde{c}_{1, j}$ is a disc of dimension $(k-1)(n+1)+1$ whose interior lays inside $\tilde{K} * \partial \tilde{L}-\tilde{L}_{((k-1)(n+1))}^{\prime}$, while the boundary belongs to $\tilde{L}_{((k-1)(n+1))}^{\prime}$. By definition of the join,the interiors 
of each pair of discs $\tilde{c}_{(k-1)(n+1)+1, j}^{\prime}, \tilde{c}_{(k-1)(n+1)+1, k \neq j}^{\prime}$ are disjoint, and the intersection of the boundaries of the two discs lays in $\tilde{L}_{((k-1)(n+1))}^{\prime}$. The $\tilde{c}_{(k-1)(n+1)+1, j}^{\prime}$ are, in fact, blocks of cells of dimensions up to $(k-1)(n+1)+1$ of $\tilde{C}$. The discs $\tilde{c}_{(k-1)(n+1)+1, j}^{\prime}$ are the $k_{2}(k-1)(n+1)+1$ cells of $\tilde{L}^{\prime}$. They are attached to the $(k-1)(n+1)$ skeleton by the definition of the join. The $(k-1)(n+1)+1$ skeleton of $\tilde{L}^{\prime}$ is $\tilde{L}_{((k-1)(n+1))}^{\prime}=\bigcup \tilde{c}_{(k-1)(n+1)+1, j}^{\prime}$.

This procedure continues up to the dimension $k(n+1)-1$ and gives a complex of dimension $k(n+1)-1$ that has the same realization of $\tilde{C}$ and thus the desired new cell decomposition $\tilde{L}^{\prime}$ of $S^{(k-1)(n+1)-1} * \mathcal{F}$.

Since the $\tilde{c}_{q, j}$ are lifts of the $c_{q, j}$, it follows that $\tilde{K}^{\prime}=\pi \tilde{L}^{\prime}$ has the same realization as $\tilde{B}$. This completes the proof.

Applying the lemma, we see that the fundamental domain of the action of $D_{2^{t}(2 h+1)}$ on $S^{4 n-1}$ via any representation $\pi_{k, l}$ follows immediately from the definition of the fundamental domain $\mathcal{F}$ given in Proposition 3.6. In a similar way, the equivariant cell decomposition of $S^{4 n-1}$ follows by that of $S^{3}$ given in Propositions 3.9 and 3.10. The unique point to be completed is the boundary of the cell $\tilde{c}_{4 q, 1}, q>0$. But this follows easily considering that $\tilde{c}_{4 q, 1}=S^{4 q-1} * \tilde{c}_{4 q-1,1}$, and hence its boundary is given by the collection of all the cells of $S^{4 q-1}$, i.e., all the orbits of $\pi$. All details can be found in the chain complex, which is explicitly given in the next section.

\section{The chain complex}

We are now in the position of giving the chain complex of $\mathbb{Z} D_{2^{t}(2 h+1)}$ modules for the split metacyclic spherical space forms $\mathbf{S}_{2^{t}(2 h+1) ; \mathbf{k} ; \mathbf{l}}^{4 n-1}=S^{4 n-1} / \pi_{\mathbf{k} ; \mathbf{l}}\left(D_{2^{t}(2 h+1)}\right)$.

Following standard notation in algebraic topology, we will denote by $C(\tilde{K}, \mathbb{Z} \pi)$ the integer chain complex of the universal covering space of a finite complex $K$ with the action of the fundamental group acting by covering transformations. This is a complex of free finitely generated modules over $\mathbb{Z} \pi$.

Theorem 5.1. The chain complex $C\left(\tilde{\mathbf{S}}_{2^{t}(2 h+1) ; \mathbf{k} ; \mathbf{l}}^{4 n-1}, \mathbb{Z} D_{2^{t}(2 h+1)}\right)$ of the universal covering space of the split metacyclic spherical space forms $\mathbf{S}_{2^{t}(2 h+1) ; \mathbf{k} ; 1}^{4 n-1}$, with the action of the fundamental group acting by covering transformations, is the following periodic complex of free finitely generated $\mathbb{Z} D_{2^{t}(2 h+1)}$ modules:

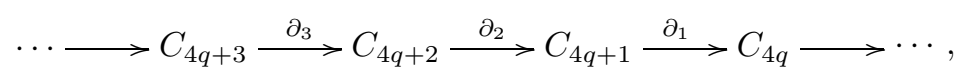

where

$$
\begin{aligned}
C_{4 q} & =\mathbb{Z} D_{2^{t}(2 h+1)}\left[\mathbf{c}_{4 q}\right], \\
C_{4 q+1} & =\mathbb{Z} D_{2^{t}(2 h+1)}\left[\mathbf{c}_{4 q+1,1}, \mathbf{c}_{4 q+1,2}, \mathbf{c}_{4 q+1,3}\right], \\
C_{4 q+2} & =\mathbb{Z} D_{2^{t}(2 h+1)}\left[\mathbf{c}_{4 q+2,1}, \mathbf{c}_{4 q+2,2}, \mathbf{c}_{4 q+2,3}\right], \\
C_{4 q+3} & =\mathbb{Z} D_{2^{t}(2 h+1)}\left[\mathbf{c}_{4 q+3}\right] .
\end{aligned}
$$

The boundaries are $\partial_{0}=0$ and 


$$
\begin{aligned}
& \partial_{4 q}\left(\mathbf{c}_{4 q}\right)=\left(1+x+\cdots+x^{2 h}\right)\left(1+y+\cdots+y^{2^{t}-1}\right) \mathbf{c}_{4 q-1}, \\
& \partial_{4 q+1}\left(\mathbf{c}_{4 q+1,1}\right)=\left(x^{\alpha} y^{-2 \beta}-1\right) \mathbf{c}_{4 q}, \\
& \partial_{4 q+1}\left(\mathbf{c}_{4 q+1,2}\right)=\left(\left(x^{\alpha} y^{\beta}\right)^{z+2^{t-2}} y^{-1}-1\right) \mathbf{c}_{4 q} \text {, } \\
& \partial_{4 q+1}\left(\mathbf{c}_{4 q+1,3}\right)=\left(y^{2 \beta z-1}-1\right) \mathbf{c}_{4 q} \text {. } \\
& \text { If } 2 h+1>2^{t-2} \text {, } \\
& \partial_{4 q+2}\left(\mathbf{c}_{4 q+2,1}\right)=y^{2 \beta z-1}\left(\sum_{s=1}^{2^{t-2}}\left(x^{\alpha} y^{2 \beta}\right)^{s} \mathbf{c}_{4 q+1,1}-\left(x^{\alpha} y^{2 \beta}\right)^{2^{t-2}} \mathbf{c}_{4 q+1,2}+\mathbf{c}_{4 q+1,3}\right), \\
& \partial_{4 q+2}\left(\mathbf{c}_{4 q+2,2}\right)=\sum_{s=2 h+1-2^{t-2}}^{2 h+1}\left(x^{\alpha} y^{-2 \beta}\right)^{s} \mathbf{c}_{4 q+1,1}+y^{2 \beta z-1}\left(\mathbf{c}_{4 q+1,2}-\mathbf{c}_{4 q+1,3}\right) \text {, } \\
& \partial_{4 q+2}\left(\mathbf{c}_{4 q+2,3}\right)=\sum_{s=0}^{2 h-2^{t-2}}\left(x^{\alpha} y^{-2 \beta}\right)^{s} \mathbf{c}_{4 q+1,1}-y^{2 \beta z-1} \mathbf{c}_{4 q+1,2}-\mathbf{c}_{4 q+1,3}, \\
& \partial_{4 q+3}\left(\mathbf{c}_{4 q+3}\right)=\left(1-\left(x^{2^{t-2}} y^{2 \beta z+2^{t-2}-1}\right)^{-1}\right) \mathbf{c}_{4 q+2,1}+\left(1-y^{1-2 \beta z}\right) \mathbf{c}_{4 q+2,2} \\
& +\left(1-\left(x^{\alpha} y^{-2 \beta}\right)^{2^{t-2}}\right) \mathbf{c}_{4 q+2,3} \text {. } \\
& \partial_{4 q+2}\left(\mathbf{c}_{4 q+2,1}\right)=\sum_{s=2^{t-2}-2 h}^{2^{t-2}}\left(x^{\alpha} y^{2 \beta}\right)^{s} y^{2 \beta z-1} \mathbf{c}_{4 q+1,1}-\left(x^{\alpha} y^{2 \beta}\right)^{2^{t-2}} y^{2 \beta z-1} \mathbf{c}_{4 q+1,2} \\
& +y^{-2 \beta(2 h+1)} \mathbf{c}_{4 q+1,3}, \\
& \partial_{4 q+2}\left(\mathbf{c}_{4 q+2,2}\right)=\sum_{s=1}^{2^{t-2}-(2 h+1)}\left(x^{\alpha} y^{2 \beta}\right)^{s} y^{2 \beta z-1} \mathbf{c}_{4 q+1,1}+y^{-2 \beta(2 h+1)} \mathbf{c}_{4 q+1,2} \\
& -y^{2 \beta z-1} \mathbf{c}_{4 q+1,3} \text {, } \\
& \partial_{4 q+2}\left(\mathbf{c}_{4 q+2,3}\right)=\sum_{s=0}^{2 h}\left(x^{\alpha} y^{-2 \beta}\right)^{s} \mathbf{c}_{4 q+1,1}-\left(y^{2 \beta z-1}+1\right) \mathbf{c}_{4 q+1,3}, \\
& \partial_{4 q+3}\left(\mathbf{c}_{4 q+3}\right)=\left(1-\left(x^{2^{t-2}} y^{2 \beta z+2^{t-2}-1}\right)^{-1}\right) \mathbf{c}_{4 q+2,1}+\left(1-y^{2 \beta(2 h+1)}\right) \mathbf{c}_{4 q+2,2} \\
& +\left(1-y^{-2 \beta z+1}\right) \mathbf{c}_{4 q+2,3},
\end{aligned}
$$

here $(\alpha, \beta)$ is a pair of integers satisfying equation (3.8), and $z=m(2 h+1)$, with $l=$ $2 m+1$. This complex is exact in all middle dimensions, namely $\operatorname{Im}\left(\partial_{j+1}\right)=\operatorname{ker}\left(\partial_{j}\right)$, for $0<j<4 n-1$.

Remark 5.2. One can show by direct calculations that the complex in Theorem 5.1 is semi-exact.

It is clear that taking the augmentation of the complex described in Theorem 5.1 we obtain a 4-periodic free resolution of $\mathbb{Z}$ over $D_{2^{t}(2 h+1)}$. This result should be compared with the ones given in [12, Chap. 7] (on which [11] is based), which has a larger rank 
in all dimensions. On the other side, we note that the resolution in Theorem 5.1 is not the minimal one, since according to the work of $O$. Talelli $[\mathbf{1 0}]$, a minimal resolution for $\mathbb{Z}$ over the split metacyclic groups has two generators in dimensions $4 q-2$ and $4 q-1$. Also note that, by applying the method of Gruenberg [5], we obtain a resolution with one generator in dimension 0 , two generators in dimension 1 and three generators in dimension 2 (see, for example, $[\mathbf{4}, 4.6]$ ). With some algebraic manipulations we could modify the boundaries given in Theorem 5.1 in such a way that two of the boundaries of the 1-dimensional chains coincide with the boundaries obtained by the Gruenberg resolution. However, we are not able to proceed further in this direction.

\section{References}

[1] A. Adem and J.F. Davis, Topics in transformation groups, in Handbook of geometric topology, 1-54, North-Holland, Amsterdam, 2002.

[2] H. Cartan and S. Eilenberg, Homological algebra, Princeton University Press, Princeton, NJ, 1956.

[3] M.M. Cohen, A course in simple homotopy theory, Grad. Texts Math. 10, Springer-Verlag, New York, 1973.

[4] E.T. Costa, O. Manzoli Neto and M. Spreafico, Vector bundles over threedimensional spherical space forms, Int. J. Math. Math. Sci. 2006 (2006), Art. ID $47574,1-11$.

[5] K.W. Gruenberg, Resolutions by relations, J. London Math. Soc. 35 (1960), 481-494.

[6] L.L. Fêmina, Ph.D. thesis, 2011, University of São Paulo. http:// www.teses .usp.br/teses/disponiveis/55/55135/tde-13012012-102038/.

[7] O. Manzoli Neto, T. de Melo and M. Spreafico, Cellular decomposition of quaternionic spherical space forms, Geom. Dedicata 162 (2013), no. 1, 9-24.

[8] J. Milnor, Whitehead torsion, Bull. Amer. Math. Soc. 72 (1966), 358-426.

[9] R.G. Swan, Periodic resolutions for finite groups, Ann. of Math. 72 (1960), no. 2, 267-291.

[10] O. Talelli, On minimal resolutions for metacyclic groups with periodic cohomology, Comm. Alg. 12 (1984), no. 11-12, 1343-1360.

[11] S. Tomoda and P. Zvengrowski, Remarks on the cohomology of finite fundamental groups of 3-manifolds, Geometry and Topology Monogr. 14 (2008), 519-556, Geom. Topol. Publ., Coventry.

[12] S. Tomoda, Cohomology rings of certain 4-periodic finite groups, Ph.D. thesis, University of Calgary, 2005.

[13] J.A. Wolf, Spaces of constant curvature, McGraw-Hill Book Co., New York, 1967.

[14] B. Zimmermann, On the classification of finite groups acting on homology 3-spheres, Pacific J. Math. 217 (2004), no. 2, 387-395. 


\section{L.L. Fêmina ligialf@famat.ufu.br}

Mathematics Faculty, UFU, Federal University of Uberlândia, Av. João Naves de Avila, 2121, Santa Mônica, CEP: 38.408-100, Uberlândia, MG, Brazil

A.P.T. Galves atrunajo@gmail.com

ICMC - Institute of Mathematics and Computer Science, USP, University of São Paulo, Av. Trabalhador são-carlense, 400, Centro, CEP: 13566-590, São Carlos, SP, Brazil

O. Manzoli Neto ozimneto@icmc.usp.br

ICMC - Institute of Mathematics and Computer Science, USP, University of São Paulo, Av. Trabalhador são-carlense, 400, Centro, CEP: 13566-590, São Carlos, SP, Brazil

M. Spreafico mauros@icmc.usp.br

ICMC - Institute of Mathematics and Computer Science, USP, University of São Paulo, Av. Trabalhador são-carlense, 400, Centro, CEP: 13566-590, São Carlos, SP, Brazil 